\title{
Fracture Mechanisms of Glass Particles under Dynamic Compression
}

Niranjan D. Parab a, Zherui Guo a, Matthew C. Hudspeth a, Benjamin J. Claus a, Kamel Fezzaa

b, Tao Sun ${ }^{b}$, Weinong W. Chen ${ }^{a, c, *}$

a School of Aeronautics and Astronautics, Purdue University, 701 West Stadium Avenue, West Lafayette, IN, USA 47907

b Advanced Photon Source, Argonne National Laboratory, 9700 South Cass Avenue, Argonne, IL, USA 60439

c School of Materials Engineering, Purdue University, 701 West Stadium Avenue, West Lafayette, IN, USA 47907

*Corresponding Author, Email: wchen@purdue.edu, Telephone No: 7654941788

\section{Abstract:}

In this study, dynamic fracture mechanisms of single and contacting spherical glass particles were observed using high speed synchrotron X-ray phase contrast imaging. A modified Kolsky bar setup was used to apply controlled dynamic compressive loading on the soda-lime glass particles. Four different configurations of particle arrangements with one, two, three, and five particles were studied. In single particle experiments, cracking initiated near the contact area between the particle and the platen, subsequently fragmenting the particle in many small sub-particles. In multi-particle experiments, a crack was observed to initiate from the point just outside the contact area between two particles. The initiated crack propagated at an angle to the horizontal loading direction, resulting in separation of a fragment. However, this fragment separation did not affect the ability of the particle to withstand further contact loading. On further compression, large number of cracks initiated in the particle with the highest number of particle-particle contacts near

(C) 2017. This manuscript version is made available under the Elsevier user license http://www.elsevier.com/open-access/userlicense/1.0/ 
one of the particle-particle contacts. The initiated cracks roughly followed the lines joining the contact points. Subsequently, the initiated cracks along with the newly developed subcracks bifurcated rapidly as they propagated through the particle and fractured the particle explosively into many small fragments, leaving the other particles nearly intact.

Keywords: Particle fracture and fragmentation, High speed X-ray phase contrast imaging, Kolsky bar, dynamic fracture

\section{Introduction}

Particles constituting granular systems undergo dynamic compression during processing, handling, transportation and usage. In many particle size reduction processes, dynamic compressive loading is applied on the particles. High speed penetration into granular materials such as sandbags imparts compressive loading on particles in front of the projectile. For the granular systems composed of brittle materials, particles fracture when loaded with sufficiently high compressive stresses. It is important to study the fracture behavior of particles under compression to improve the efficiency of the processes where particle fracture is desired such as particle milling process. In some cases, particle fracture can result in small sub-particles which can be harmful to equipment and personnel. Silica dust caused by fracture of silica particles can cause incurable lung diseases such as silicosis and lung cancer [1]. In such scenarios, understanding of the particle fracture process will help in preventing the conditions which cause fracture and hence improve working environments. 
In early works aimed at studying the deformation and fracture processes of single particles, a particle composed of a brittle material was loaded in quasi-static compression between two stiff platens (diametral compression) and fracture forces were recorded [2]-[7]. For glass particles, researchers have fitted the Weibull distribution to the fracture forces to study it statistically [2], [8]. Two different experimental methodologies have been used to study the behavior of particles under dynamic compression: single particle impact and drop tower testing. In single particle impact experiments, a single particle was impacted against a rigid target and the fracture process was studied using various in-situ and postprocessing methods [5], [9]-[15]. It was observed that a critical threshold velocity was required to induce fracture in glass particle [10]. Below the threshold velocity, the particles bounced back after the impact. Above the threshold velocity, the particles were observed to fragment explosively [10]. In drop tower testing, a predetermined weight was dropped on a single particle sandwiched between two rigid platens [16]-[19]. The particle fracture mode was observed to depend on the material properties of the particle and the energy input into the particle [12], [19]. Fragments increased in number and decreased in size as the input energy was increased for ceramic particles [12], [19].

An analytical formulation for the stress states present in a single particle under diametral compression and impact loading has been proposed [3], [19], [20]. Based on this stress state, two conflicting parameters were proposed as the critical factors causing fracture of particles. Some researchers reported the maximum tensile stress inside the volume of the particle as the critical stress causing the fracture of the particle [2], [20], [21]. In contrast, the maximum tensile stress just outside the contact area between the particle and the platen was also proposed as the critical stress leading to fracture [22]. As such, an 
unambiguous criterion for particle fracture will be helpful in planning for various processes under different loadings. Furthermore, in granular systems, both particleparticle and particle-boundary contacts affect the overall behavior of the system under loading. The aforementioned single particle studies are limited to the particle-plane contact and do not consider the effects of particle-particle contact condition on the deformation and fracture of the particle. Hence the high speed X-ray Phase Contrast Imaging (PCI) setup synchronized with a modified Kolsky bar apparatus was used to investigate the fracture of single and contacting glass particles under dynamic compression. The aim of this study is to assess the effects of contact conditions on the fracture mechanisms of glass particles.

\section{Materials and Methods}

\subsection{Glass Particles}

Commercially available solid spherical soda lime glass particles (Cospheric LLC, Santa Barbara, CA) of diameters between 1.00 to $1.18 \mathrm{~mm}$ were used. Some glass particles were annealed to study the effects of residual stresses on the fracture behavior. Glass particles were heated to the temperature of $540^{\circ} \mathrm{C}$ at a rate of $20^{\circ} \mathrm{C} / \mathrm{min}$ and then were maintained at that temperature for 4 hours. The furnace was then turned off and the particles were allowed to return to room temperature at the nominal cooling rate of $4^{\circ} \mathrm{C} / \mathrm{min}$. The rate of cooling was not controlled. For each experiment, particles were randomly selected from a large collection of particles with aforementioned diameters. For contacting particle experiments, all included particles were either untreated or annealed (i.e. each experiment contained solely as-received or solely annealed particles). Glass particles were chosen in this study as model particles because they display characteristic elastic-brittle response. 
Furthermore, they were easy to obtain commercially in nominally perfect spherical shapes, thus reducing the complexity in the analysis introduced by irregularly shaped particles.

\subsection{Experimental method}

A modified Kolsky bar setup was used to apply the controlled compressive loading on the particles and the high speed PCI was used to record the in-situ fracture mechanisms in the particles. This experimental method has been used to study the failure mechanisms of various brittle materials including glass plates [23], sand particles [24], and polycrystalline silicon micro-beams [25]. More details of the experimental procedure are provided elsewhere [25]. The schematic of the experimental setup is presented in Figure 1.

Polychromatic, high intensity X-ray PCI measurements were performed at beam line 32-IDB, Advanced Photon Source (APS), Argonne National Laboratory, Argonne, Illinois, USA. In a synchrotron X-ray source, electron bunches are maintained in a circular storage ring and emit X-ray radiation in the tangential direction. The brilliance of the X-rays is further increased by using an undulator in a storage ring [26]. The fundamental energy of the X-ray beam at the beamline 32-ID with the undulator gap set to $11 \mathrm{~mm}$ was peaked at $23.6 \mathrm{KeV}$. The size of the X-ray beam on the sample was $2560 \times 1600 \mu \mathrm{m}^{2}$. The X-ray intensity on the sample was approximately $4.0 \times 10^{16}$ photons $/ \mathrm{s} / \mathrm{mm}^{2} / 0.1 \%$ BW. X-ray PCI employs the change in the phase of the X-rays as they pass through the sample containing materials with different refractive indices in order to obtain high contrast images. The changes in refractive index resulting from phase changes provide greater edge contrast which is beneficial for visualizing cracks in the materials [27], [28]. In current study, inline holographic technique was used to obtain the requisite X-ray phase contrast [29]. A single 
crystal $\mathrm{Lu}_{3} \mathrm{Al}_{5} \mathrm{O}_{12}:$ Ce scintillator (dimensions: $10 \mathrm{~mm}$ x $10 \mathrm{~mm}$ x $100 \mu \mathrm{m}$ ) was used to convert the propagated X-ray signal to visible light wavelengths. The converted images were recorded using an ultra-high speed camera (Shimadzu Hyper Vision HPV-X2, Tokyo, Japan). The temporal resolution of the recorded images was $200 \mathrm{~ns}$ or $500 \mathrm{~ns}$ ( 5 million or 2 million frames per second). Exposure times of $110 \mathrm{~ns}$ and $200 \mathrm{~ns}$ were used for the temporal resolution of $200 \mathrm{~ns}$ and $500 \mathrm{~ns}$ respectively. The resolution of the imaging system was estimated to be $6.4 \mu \mathrm{m} /$ pixels and the frame size was 102,000 pixels ( $400 \mathrm{px} \mathrm{X}$ $256 \mathrm{px})$.

A Kolsky bar apparatus is commonly used to characterize the material behavior at high strain rates $\left(10^{2}-10^{5} \mathrm{~s}^{-1}\right)$ [30], [31]. For the current experiments, a miniature compression Kolsky bar was used [25]. The Kolsky bar was composed of a striker bar $(\varnothing=12.7 \mathrm{~mm}$, length $=305 \mathrm{~mm})$ and an incident bar $(\varnothing=12.7 \mathrm{~mm}$, length $=1372 \mathrm{~mm})$, both manufactured from a high strength steel alloy. The transmission bar in the conventional Kolsky bar apparatus was replaced with a load cell (Kistler 9212, Winterhur, Switzerland) mounted on a heavy aluminum backstop due to space constraints in the APS X-ray hutch. Two semi-conductor strain gauges (Kyowa KSP-2-1K-E4, Chofu, Japan) were attached diametrically to the surface of the incident bar and were connected in a half Wheatstone bridge configuration. The strain gauge assembly was used to record both the incident and reflected pulses. The load cell was used to record the force response of particles at the back end. Both the strain gauge signal and load signal were synchronized and collected via an oscilloscope (Tektronix DP07104C, Beaverton, Oregon). The velocity at the bar end was calculated from the recorded strain signals using Eq. 1. 


$$
v(t)=C_{B} \times\left(\epsilon_{i}(t)-\epsilon_{r}(t)\right)
$$

Where $C_{B}$ represents the elastic wave velocity in the bar. $\varepsilon_{i}$ and $\varepsilon_{r}$ represent the incident and reflected strain values respectively. The bar end displacement was then calculated using Eq. 2.

$$
d(t)=\int_{o}^{t} v(\tau) d \tau
$$

In single particle experiments, the back pin was observed to displace significantly due to the compliance in the setup. In that case, the relative displacement values between the two pins were calculated directly from the recorded high speed images.

Four different particle arrangements were investigated comprising of one, two, three, and five particles respectively. A schematic of the particle arrangements is presented in Figure 2. For single particle experiments, the particle was constrained in an aluminum holder and compressed between two steel pins. For two particle experiments, the particles were constrained in an aluminum holder with the same dimensions as the ones used in the single particle experiments. A steel pin was used to compress the particles against each other and against the holder. For three and five particle experiments, the particles were sandwiched between two $1.0 \mathrm{~mm}$ thick PMMA plates separated by a $1.1 \mathrm{~mm}$ thick steel plate. A $2.2 \mathrm{~mm}$ wide channel was machined in the steel plate and the particles were placed in this channel. A $2.2 \mathrm{~mm}$ wide flat faced steel plate was used to compress the particles against each other. In all experiments, the sample holders were placed between the bar end and the load cell and the compressing mechanism (steel pin or steel plate) was mounted on the bar end. Due 
to the limitations in the particle holder fixtures, the force response was not recorded for the three and five particle experiments.

A typical experiment started with a manual signal which triggered the gas gun and launched the striker bar towards one end of the incident bar. The impact of the striker bar with the incident bar generated a compression stress wave in the incident bar. This stress wave propagated to the other end of the bar where it pushed the compressing mechanism onto the particle arrangement, thus compressing the specimens at a constant velocity of around $6 \mathrm{~ms}^{-1}$. The stress wave was sensed by the strain gauges as it propagated along the bar. The sensing of the stress wave also provided the trigger signal for the X-ray shuttering system and the high speed imaging system. Appropriate delay times were set for both shuttering and imaging systems to account for the time of propagation for the wave from strain gauge location to the end of the incident bar. The high intensity X-ray beam passed through the specimen as it was being compressed. The deformation and fracture processes were recorded using the scintillator-camera system. After the experiment, the brightness and contrast in the recorded high speed images were adjusted using an image processing software: ImageJ [32]. Further, a colorization scheme was applied (specifically: 'look-up table: Fire') to help with a better visualization of cracking and damage in the specimens. Each experiment was repeated at least three times and the fracture mechanisms were observed to be consistent for the repeated experiments.

There are several advantages of using the high speed synchrotron X-ray imaging setup for capturing the fracture mechanisms of glass particles. Since the X-ray images are obtained as projected images on the scintillator plane, the blurring of some parts of the particles due 
to small depth of focus in optical imaging is avoided. Previously, researchers have used refractive index matching with a surrounding fluid to observe the fracture of spheres more clearly [18], [33]. However, the surrounding fluid may apply confining forces on the spheres which may alter the stress state in the sphere. Since high intensity X-rays can pass through common engineering materials such as aluminum, the holders for multi-particle experiments can be manufactured from various different materials and are not restricted by the transparency requirement. Further, although the high speed X-ray imaging technique is used for transparent glass particles here, it can also be utilized to investigate the sub-surface damage and fracture mechanisms in opaque particles which will be impossible using the conventional optical high speed imaging.

For the one and two particle experiments, the Hertzian force-displacement relations for the linear elastic materials were compared against the experimental force-displacement data. For one particle compression, which involves two sphere-flat plate contacts, the forcedisplacement relation is given by eq. (3).

$$
\begin{gathered}
F=\frac{2}{3} E_{\text {steel,glass }}^{*} \sqrt{R} d^{\frac{3}{2}} \\
\text { Where } E^{*} \text { is given by: } \\
\frac{1}{E_{\text {steel,glass }}^{*}}=\frac{1-v_{\text {glass }}^{2}}{E_{\text {glass }}}+\frac{1-v_{\text {steel }}^{2}}{E_{\text {steel }}}
\end{gathered}
$$

Where, $R$ is the radius of the particle, $F$ is the force at the back-end, and $d$ is the relative displacement between the flat plates. 
For two particle experiments, three different contacts were considered. First, the sphereflat plates contact between the particle (glass) and pin (steel). Second, the sphere-sphere contact between two particles (glass), and finally, the sphere-flat plate contact between the particle (glass) and the holder (aluminum). The force-displacement relation is given by eq. (5).

$$
d=\left[\frac{\frac{3}{4} F}{E_{\text {steel,glass }}^{*} \sqrt{R}}\right]^{\frac{2}{3}}+\left[\frac{\frac{3}{2 \sqrt{2}} F}{E_{\text {glass, } \text { glass }}^{*} \sqrt{R}}\right]^{\frac{2}{3}}+\left[\frac{\frac{3}{4} F}{E_{\text {glass,aluminum }}^{*} \sqrt{R}}\right]^{\frac{2}{3}}
$$

The material properties for steel, glass, and aluminum are provided in Table 1.

\section{Results}

\subsection{Single particle experiments}

The high speed images with the corresponding force-displacement plots for untreated and annealed single particle experiments are presented in Figures 3 and 4 respectively. In both experiments, a single glass particle was compressed between two hardened steel pins. As the input stress wave reached the bar end and the pin attached to the incident bar end started to move, the particle was gradually compressed between the pins. Significant backward movement of the back pin was observed and hence eq. 2 did not give an accurate estimate of the particle deformation. Therefore, particle deformation was measured from the separation between the pins from the high speed images. In the force-displacement curves, each black circle represents one frame; separation between consecutive circles is 10 frames or $5 \mu \mathrm{s}$. For the untreated particle, the movement of the pin first elastically compressed the particle. When the compressive force reached the critical value, multiple 
meridional cracks were observed to initiate near one of the particle-pin contacts. The meridional cracks propagated through the particle towards the other particle-pin contact. The cracks bifurcated as they propagated through the particle, thus explosively fragmenting the particle. The fracture mechanism for the annealed particles was very similar to the one observed for the untreated particle.

The force-displacement data matched the Hertzian relation for both untreated and annealed particles for small displacements. The force value was observed to increase until the explosive fragmentation of the particle. The experiments were repeated three times for both untreated and annealed particles. The observed failure mechanisms were consistent across the repeated experiments. The observed failure force was $875.0 \pm 207.6 \mathrm{~N}$ from three repeated experiments for the untreated particles and $664.0 \pm 345.9 \mathrm{~N}$ from three repeated experiments for the annealed particles.

\subsection{Two particle experiments}

The force-displacement plots along with the corresponding high speed images from untreated and annealed two particle experiments are presented in Figures 5 and 6 respectively. For the untreated particles, as the pin started moving, particles first rearranged slightly such that good contact was established between pin-particle, particleparticle, and particle-holder. Once all the components were in good contact, particles began to deform elastically. During the elastic deformation, the contact area between the particles gradually increased.

Eventually, the stresses at the point just outside the particle-particle contact area reached the critical value and an angular crack initiated at that point in one of the particles. This 
crack propagated at approximate angle of $65^{\circ}$ to the loading direction and terminated at the surface. This separated a fragment from the particle. Even after the initiation of the angular crack and the separation of the fragment, the measured force continued to further increase.

As the particles were even further compressed, a network of cracks developed inside one of the particles near the particle-particle contact initiating fracture of that particular particle. These cracks then propagated away from the contact site, rapidly bifurcating as they moved in the particle, hence explosively fracturing the particle into many small pieces. It is important to note that as the first particle fractured explosively, only minor damage was observed in the second particle. The observed fracture mechanisms were similar for the annealed particles. The experiment was repeated 15 times for the untreated particles and three times for the annealed particles and the observed fracture behavior was very similar for the repeated experiments.

The experimental force-displacement curve matched the Hertzian contact fit for small deformations, though, a slight shift from the Hertzian fit was observed after the angular crack was initiated in the particle. It is important to note that the particles could withstand the loading even after the separation of the fragment from the angular cracks. The ratio between the forces at the initiation of angular cracking to the force at complete fragmentation was $0.35 \pm 0.16$. The force at the backend dropped only when one of the particles fragmented explosively and hence the peak force corresponding to the explosive fragmentation was defined as the failure force. A large variation in failure force was observed in the experiments. The observed failure force was $339.3 \pm 70.3 \mathrm{~N}$ from 15 
repeated experiments for untreated particles and 298.6 $\pm 60.9 \mathrm{~N}$ from three repeated experiments for annealed particles.

\subsection{Three particle experiments}

Three particle experiments were performed to further investigate the effects of contact conditions on the fracture behavior of the glass particles. The recorded high speed X-ray images of the fracture behavior of three contacting particles under dynamic compression from a representative experiment are presented in Figure 7. As the particles were compressed against each other, good contact was formed between the single particle close to the bar end and two particles away from the bar end. Fragment separation was observed at one of the particle-particle contacts as the particles were compressed further. The cracking that led to the particle fracture initiated near the particle-particle contacts in the particle with two particle-particle contacts and rapidly propagated towards the particleplate contact. A large number of sub-cracks initiated in the particle as the first crack propagated through the particle. The direction of the sub-cracks was also along the line joining the contact points. Cracking and fragmentation was also observed along the two particle-particle contacts. Ultimately, the particle fragmented explosively in large number of small fragments. There was no damage or cracking observed in two other particles. The experiment was repeated three times and repeated experiments displayed very similar fracture behavior.

\subsection{Five particle experiments}

The high speed X-ray images from a representative five particle compression experiment are presented in Figure 8. Contact fragment separation was observed at two contacts in the 
middle particle (four particle-particle contacts). Along with the contact fragment separation, the cracking that led to the ultimate fracture initiated as a meridional crack in the center and two cracks at an angle of approximately $45^{\circ}$ to the meridional crack in the direction of the particle-particle contacts in the middle particle. On further compression, two more cracks in the directions of other two particle-particle contacts initiated. A large number of sub-cracks developed concurrently and ultimately, the middle particle fragmented explosively from bifurcation of initial cracks and sub-cracks. No damage was observed in any other particles. In three repeated experiments, very similar fracture mechanisms were observed.

\section{Discussion}

The effects of contact conditions on the fracture behavior of the glass particles under dynamic compression were studied using the synchronized Kolsky bar - high speed X-ray imaging system. Different crack initiation points were identified for different contact conditions. However, the ultimate failure mode (explosive fragmentation) was similar for all contact conditions.

The force-displacement curves for single and two particle compression experiments matched the linear elastic Hertzian contact solution for small displacements. The good match between the experimental measurements and the Hertzian solution suggests that the material behavior of glass is elastic and displays a brittle behavior. For both experiments, some discrepancy was observed between the experimental measurements and the analytical solution for large displacements. The displacement at the fracture point for both the experiments was less than the $10 \%$ of the particle diameters which is widely 
believed to be the limit for the Hertzian contact solution [34]. Hence, discrepancy may be due to small variations in the material properties of the glass particles [35]. Further, for the single particle experiments, the relative displacements were manually measured from the high speed images. The manual method may have introduced an error of 1 to 2 pixels (approximately 1\% of diameter) in displacement measurements. Further, slight reorientation in the out-of-plane direction (normal to the plane of the scintillator), may have further contributed to the error in the measurements. For the two particle experiments, the initial cracking at the particle-particle contact may have caused deviation from the ideal Hertzian contact behavior.

The initiation of cracking was observed near one of the particle-plate contact points inside the volume for the single particle compression. The cracking started near the particleparticle contact for the multi-particle experiments in the form of a Hertzian cone crack. This crack initiation behavior matches the previously reported fracture initiation behavior deduced from the post-mortem analysis in glass particles [36]. The observed fracture behavior supports the use of tensile stress inside the volume of the particle as the critical parameter governing fracture for the single particle experiments, as previously proposed by various researchers [2], [20]. For the two particle compression experiments, the tensile stress just outside of the contact area is higher for the sphere-sphere contact than that for the sphere-flat plate contact according to the Hertzian contact theory for the linear elastic materials (Appendix A1). Further, for reasonable flaw size in the particle, the compressive force required to initiate cracking at the particle-particle contact is lower than that required to initiate cracking at the particle-boundary contact and inside the volume of the particle (Appendix A2). Hence, the experimentally observed location of crack initiation at 
the particle-particle contact is consistent with the theoretically predicted crack initiation location.

Once the crack started at the particle-particle contact, it quickly propagated at an angle to the horizontal loading direction in the two particle experiments. The angle between crack and the horizontal was observed to be around $65^{\circ}$. Similar angular cracks, commonly called as Hertzian cone cracks, have been observed in flat plates impacted with spherical projectiles [37]-[39]. The angle of cone cracks for the impacted soda lime glass plates was observed to be around $62.5^{\circ}[39]$. The change in geometry of the experiment from sphereflat plate contact to sphere-sphere contact may explain the slight mismatch in cone crack angles. It is further postulated that the angle of cone cracking is dictated by the path of the crack that leads to maximum release of the strain energy as the crack propagates [40]. The angular crack quickly propagated to the surface of the particle, thus separating a fragment near the particle-particle contact. Observation of fragment separation near the contact point for quasi-static compression of glass spheres has been reported previously [33]. However, fragment separation was observed only in multi-particle experiments in the current study. The ability of the particle to withstand load was not diminished significantly even after the separation of the fragment, as the force at the back-end continued to increase.

From the high speed X-ray images, it can be observed that the cracks leading to the complete fracture of the particles initiated inside the volume of the particle near the particle-particle contact for two particle experiments. It is postulated that the oscillations from the initial cracks create tension as high as the original compression which give rise to 
the cracking of the particle [33]. However, the delay between the initiation of angular cracks and cracks inside the volume was significantly greater than the time required for the release waves from the angular cracks to travel inside the particles. The initiated cracks bifurcated rapidly as they propagated through the particle. Along with the rapid bifurcation of the initial cracks, large number of sub-cracks also developed and propagated through the particle. The development of sub-cracks and subsequent rapid bifurcation resulted in the particle fragmenting explosively. The propagation of cracks through the particle was similar to previously reported propagation of fracture wave through glass structures [41][43]. For the two particle experiments, the choice of the particle that fractured was random (in some experiments, the particle close to the pin fractured first. In others, the particle away from the pin fractured first). Hence, the order of the fracture may be governed by the internal flaws present in each particle. Furthermore, slight variation in the loading geometries may have also contributed to randomness in the fracture order for the particles. However, the variations in the stress state from the changes in the loading geometry were not large enough to change the fracture modes.

For the three and five particle experiments, initial fragment separation at the particleparticle contact was also observed for the particle with the highest number of particleparticle contacts. Further, cracks developed in the direction of the lines joining both particle-particle and particle-plate contact points in the same particle. In the three particle experiments, the cracks initiated at the particle-particle contact. Similar cracks along the contact points were also observed in multi-contact compression of brittle cylinders [44]. The initiation of cracks in the direction of contacts is governed by the direction of the tensile stresses developed in the particles. In five particle experiments, the major 
meridional crack developed between two diametral particle-particle contacts. Due to the symmetry of the setup, the stress field must also be symmetric. Hence, the development of the meridional crack must be governed by the size and the orientation of the flaws in the particle near the location of the maximum tensile stress inside the volume of the particle. Several sub-cracks also developed in the particle following the primary cracks. The bifurcation of the cracks and the development of sub-cracks led to the explosive fragmentation of the particle with the highest number of particle-particle contacts. It should be noted here that the total number of contacts was the same for all the particles. However, the particles that did not fracture (particle with less number of particle-particle contacts) were in contact with the holder which was a case of particle-flat plate contact. As seen from two particle experiments, the particles were more susceptible to fracture initiation at the particle-particle contacts as compared to the particle-flat plate contacts. This behavior was also observed for the multi-particle experiments where the particles showed more propensities for fracture at the particle-particle contacts and the particle with the highest number of particle-particle contacts fractured first.

Although the crack initiation behavior was dependent on the contact conditions, all the particles fractured in the explosive fragmentation mode ultimately. This matches the previously reported failure mode for single glass particles under quasi-static and dynamic compressive loading [10]. The observed cracking behavior of the glass particles will be helpful in numerical modeling of particle fracture, specifically using the discrete element methods. Various researchers have proposed different techniques for replacing the fractured particles with fragments for modeling the granular systems that are susceptible to particle fracture [45]-[47]. The experimental results from this study may be used to 
qualitatively guide the particle replacement techniques for glass particles in numerical simulations. Further post-mortem quantitative analysis may be performed on the fragment size distributions to form an accurate particle replacement strategy.

The observed fracture mechanisms were similar for the untreated and annealed glass particles, which matches the previously reported results for impact fracture of single glass particles [10]. We can conclude that the observed explosive fragmentation fracture was not governed by the residual stresses present in the particle, but was the inherent fracture mechanism for the glass spheres.

\section{Conclusions}

In this study, high speed synchrotron X-ray PCI was synchronized with a modified Kolsky bar setup to record the in-situ fracture mechanism of glass particles as they were dynamically compressed. High speed X-ray PCI provides an ideal method to capture the sub-surface damage in particles. The dynamic compressive loading was applied on one, two, three, and five contacting particles to assess the effects of contact conditions on the fracture mechanisms. The experiments with one and two particles were repeated with annealed glass particles to investigate the effects of residual stresses. The cracking initiated inside the volume near the particle-plate contact for the single particle experiments. For two particle experiments, the first crack was observed to initiate in one of the particles just outside the particle-particle contact. This conical crack propagated at an angle of $65^{\circ}$ to the horizontal and terminated at the surface, thus separating a fragment from the particle. The conical crack did not erode the particles ability to withstand more loading. Similar conical cracks and subsequent fragment separations were observed near the particle-particle 
contacts for three and five particle experiments. On further compression, cracks developed in the volume of the particles with separated fragment just under the particle-particle contact. For all multi-particle experiments, the initial cracks were along the lines joining the contact points. These initiated cracks and newly developed sub-cracks bifurcated rapidly as they propagated through the particle, fracturing it into many small fragments. It can be concluded that although the initiation of fracture in particles was governed by the tensile stresses near the contact area which lead to the conical cracks, the ultimate fracture of the particles was governed by the maximum tensile stress inside the volume of the particle and not at the contact surface. The observed fracture mechanisms provide additional insight into the fracture behavior of glass particles, which will be helpful in guiding the numerical modeling efforts.

\section{Acknowledgments}

We appreciate professional help from A. Deriy with certain technical and safety aspects of our experiments at the APS. Use of the Advanced Photon Source, an Office of Science User Facility operated for the U.S. Department of Energy (DOE) Office of Science by Argonne National Laboratory, was supported by the U.S. DOE under Contract no. DE-AC0206CH11357. This research was supported by the ONR Grant N00014-14-1-0628 (Program manager: Dr. David Shifler).

\section{References}

[1] D. Rosner, "The long struggle to protect workers' lungs against silicosis," The milbank Quarterly, pp. 191-194, 2014. 
[2] B. A. Kschinka, S. Perrella, H. Nguyen, and R. C. Bradt, "Strengths of Glass Spheres in Compression," J. Am. Ceram. Soc., vol. 69, no. 6, pp. 467-472, Jun. 1986.

[3] P. H. Shipway and I. M. Hutchings, "Fracture of brittle spheres under compression and impact loading I: Elastic stress distributions," Philos. Mag. A, vol. 67, no. 6, pp. 1389-1404, 1993.

[4] O. Tsoungui, D. Vallet, J-C. Charmet, and S. Roux, "Size effects in single grain fragmentation," Granul. Matter, vol. 2, pp. 19-27, 1999.

[5] A. D. Salman and D. A. Gorham, "The fracture of glass spheres," Powder Technol., vol. 107, pp. 179-185, 2000.

[6] K. Schönert, "Breakage of spheres and circular discs," Powder Technol., vol. 143-144, pp. 2-18, 2004.

[7] P. V. Lade and H. Karimpour, "Time effects in granular materials: From micro to macro behavior," in Geomechanics from Micro to Macro, 2015, pp. 1243-1248.

[8] J. Huang, S. Xu, H. Yi, and S. Hu, "Size effect on the compression breakage strengths of glass particles," Powder Technol., vol. 268, pp. 86-94, 2014.

[9] P. H. Shipway and I. M. Hutchings, "Fracture of brittle spheres under compression and impact loading II: Results for lead-glass and sapphire spheres," Philos. Mag. A, vol. 67, no. 6, pp. 1405-1421, 1993.

[10] E. W. Andrews and K.-S. Kim, "Threshold conditions for dynamic fragmentation of glass particles," Mech. Mater., vol. 31, pp. 689-703, 1999. 
[11] L. Vogel and W. Peukert, "Breakage behavior of different materials - construction of a mastercurve for the breakage probability," Powder Technol., vol. 129, pp. 101-110, 2003.

[12] A. D. Salman, G. K. Reynolds, J. S. Fu, Y. S. Cheong, C. A. Biggs, M. J. Adams, D. A. Gorham, J. Lukenics, and M. J. Hounslow, "Descriptive classification of the impact failure modes of spherical particles," Powder Technol., vol. 143-144, pp. 19-30, 2004.

[13] M. Meier, E. John, D. Wieckhusen, W. Wirth, and W. Peukert, "Influence of mechanical properties on impact fracture: Prediction of the milling behavior of pharmaceutical powders by nanoindentation," Powder Technol., vol. 188, pp. 301-313, 2009.

[14] Y. Rozenblat, A. Levy, H. Kalman, and J. Tomas, "Impact velocity and compression force relationship - Equivalence function," Powder Technol., vol. 235, pp. 756-763, 2013.

[15] P. Mueller, S. Aman, and J. Tomas, "Evaluation of Impact Velocity and Compression Force of Moist Zeolite 4A Granules at Breakage using an Equivalence Function," Chem. Eng. Technol., vol. 37, no. 5, pp. 813-818, May 2014.

[16] L. M. Tavares and R. P. King, "Single-particle fracture under impact loading," Int. J. Miner. Process., vol. 54, pp. 1-28, 1998.

[17] K. T. Chau, X. X. Wei, R. H. C. Wong, and T. X. Yu, "Fragmentation of brittle spheres under static and dynamic compressions: Experiments and Analyses," Mech. Mater., vol. 32, pp. 543-554, 2000.

[18] M. . Chaudhri, "Impact breakage of semi-brittle spheres," Powder Technol., vol. 143- 
144, pp. 31-40, 2004.

[19] S. Z. Wu and K. T. Chau, "Dynamic response of an elastic sphere under diametral impacts," Mech. Mater., vol. 38, pp. 1039-1060, 2006.

[20] Y. Hiramatsu and Y. Oka, "Determination of the tensile strength of rock by a compression test of an irregular test piece," Int. J. Rock Mech. Min. Sci., vol. 3, pp. 8999, 1966.

[21] K. T. Chau and X. X. Wei, "Spherically isotropic, elastic spheres subject to dimetral point load strength test," Int. J. Solids Struct., vol. 36, pp. 4473-4496, 1999.

[22] R. H. Brzesowsky, C. J. Spiers, C. J. Peach, and S. J. T. Hangx, "Failure behavior of single sand grains: Theory versus experiment," J. Geophys. Res., vol. 116, p. B06205, 2011.

[23] N. D. Parab, J. T. Black, B. Claus, M. Hudspeth, J. Sun, K. Fezzaa, and W. W. Chen, “Observation of Crack Propagation in Glass Using X-ray Phase Contrast Imaging," Int. J. Appl. Glas. Sci., vol. 5, no. 4, pp. 363-373, 2014.

[24] N. D. Parab, B. Claus, M. C. Hudspeth, J. T. Black, A. Mondal, J. Sun, K. Fezza, X. Xiao, S. N. Luo, and W. W. Chen, "Experimental assessment of fracture of individual sand particles at different loading rates," Int. J. Impact Eng., vol. 68, pp. 8-14, 2014.

[25] M. Hudspeth, B. Claus, S. Dubelman, J. Black, A. Mondal, N. Parab, C. Funnell, F. Hai, M. L. Qi, S. N. Luo, and W. Chen, "High speed synchrotron x-ray phase contrast imaging of dynamic material response to split Hopkinson bar loading," Rev. Sci. Instrum., vol. 84, p. 025102:1-025102:7, 2013. 
[26] A. Balerna and S. Mobilio, "Introduction to Synchrotron Radiation," in Synchrotron Radiation basics methods and applications, 1st ed., S. Mobilio, F. Boscherini, and C. Meneghini, Eds. Berlin: Springer-Verlag, 2015, pp. 3-28.

[27] S. W. Wilkins, T. E. Gureyev, D. Gao, A. Pogany, and A. W. Stevenson, "Phase-contrast imaging using polychromatic hard X-rays," Nature, vol. 384, p. 335, 1996.

[28] R. P. Murrie, A. W. Stevenson, K. S. Morgan, A. Fouras, D. M. Paganin, and K. K. W. Siu, "Feasibility study of propagation-based phase-contrast X-ray lung imaging on the Imaging and Medical beamline at the Australian Synchrotron," J. Synchrotron Radiat., vol. 21, no. 2, pp. 430-445, Mar. 2014.

[29] P. Spanne, C. Raven, I. Snigireva, and A. Snigirev, "In-line holography and phasecontrast microtomography with high energy x-rays," Phys. Med. Biol., vol. 44, no. 3, pp. 741-749, Mar. 1999.

[30] H. Kolsky, "An investigation of the mechanical properties of materials at very high rates of loading," Proc. Phys. Soc. B, vol. 62, p. 676, 1949.

[31] W. W. Chen and B. Song, Split hopkinson (Kolsky) bar: Design, testing and applications. New York: Springer, 2011.

[32] C. A. Schneider, W. S. Rasband, and K. W. Eliceiri, "NIH Image to ImageJ: 25 years of image analysis," Nat methods, vol. 9, no. 7, pp. 671-675, 2012.

[33] K. Schonert, "Breakage of spheres and circular disks," Powder Technol., vol. 143-144, pp. 2-18, 2004. 
[34] K. L. Johnson, Contact Mechanics. New York: Cambridge University Press, 1985.

[35] M. B. Zbib, N. D. Parab, W. W. Chen, and D. F. Bahr, "New pulverization parameter derived from indentation and dynamic compression of brittle microspheres," Powder Technol., vol. 283, pp. 57-65, 2015.

[36] M. K. Gundepudi, B. V. Sankar, J. J. Mecholsky, and D. C. Clupper, "Stress analysis of brittle spheres under multiaxial loading," Powder Technol., vol. 94, no. 2, pp. 153$161,1997$.

[37] M. M. Chaudhri and C. Liangyi, "The orientation of the hertzian cone crack in sodalime glass formed by oblique dynamic and quasi-static loading with a hard sphere," J. Mater. Sci., vol. 24, pp. 3441-3448, 1989.

[38] S. Y. Oh and H. S. Shin, "Effect of particle impact velocity on cone crack shape in ceramic material," Key Eng. Mater., vol. 297, pp. 1321-1326, 2005.

[39] M.. Chaudhri, "Dynamic fracture of inorganic glasses by hard spherical and conical projectiles," Philos. Trans. R. Soc. A, vol. 373, p. 20140135, 2015.

[40] C. Kocer and R. E. Collins, "Angle of Hertzian cone cracks," J. Am. Ceram. Soc., vol. 81, no. 7, pp. 1736-1742, 1998.

[41] H. D. Espinosa, Y. Xu, and N. S. Brar, "Micromechanics of failure waves in glass: I, Experiments," J. Am. Ceram. Soc., vol. 80, no. 8, pp. 2061-2073, 1997.

[42] D. D. Radford, G. R. Willmott, S. M. Walley, and J. E. Field, "Failure mechanims in ductile and brittle materials during taylor impact," J. Phys. IV, vol. 110, pp. 687-692, 
2003.

[43] E. Strassburger, P. Patel, J. W. McCauley, C. Kovalchick, K. T. Ramesh, and D. W. Templeton, "High-speed transmission shadowgraphic and dynamic photoelasticity study of stress wave and impact damage propagation in transparent materials and laminates using edge-on impact method: Report Number: ARL-RP-203," Aberdeen Proving Ground, MD, 2008.

[44] Y. Salami, C. Dano, P.-Y. Hicher, G. Colombo, and P. Denain, "The effects of the coordination on the fragmentation of a single grain," IOP Conf. Ser. Earth Environ. Sci., vol. 26, no. 1, p. 12015, 2015.

[45] O. Tsoungui, D. Vallet, and J.-C. Charmet, "Numerical model of crushing of grains inside two-dimensional granular materials," Powder Technol., vol. 105, no. 1, pp. 190198, 1999.

[46] O. Ben-Nun and I. Einav, "The role of self-organization during confined comminution of granular materials," Philos. Trans. R. Soc. A Math. Phys. Eng. Sci., vol. 368, no. 1910, pp. 231-247, 2009.

[47] G. R. McDowell and J. P. De Bono, "On the micro mechanics of one-dimensional normal compression," Geotechnique, vol. 63, no. 11, pp. 895-908, 2013.

\section{Appendix A1: Particle-Particle interaction}

For two linear elastic bodies of revolution in contact, the maximum tensile stress at the contact is given by [34]: 


$$
\sigma_{c}=\frac{1-2 v}{3}\left[\frac{6 F E^{*^{2}}}{\pi^{3} R^{*^{2}}}\right]^{\frac{1}{3}}
$$

For the contact between sphere and the flat plane, $R^{*}$ is equal to the radius of the particle.

For the contact between two spheres, $R^{*}$ is equal to half of the radius of the particle.

Further, $E^{*}$ at the particle-steel plate contact is 1.47 times the $E^{*}$ at the particle-particle

contact. Hence, the maximum tensile stress at the sphere-sphere contact is greater than that at the sphere-flat plate contact.

\section{Appendix A2: Particle-flat plate interaction}

For a spherical particle compressed between two flat plates, the maximum tensile stress is observed just outside of the contact area between the plate and the particle. This stress is given by [22]:

$$
\begin{gathered}
\sigma_{\mathrm{c}}=\frac{1-2 v_{\mathrm{g}}}{\pi \mathrm{a}^{2}} \\
a=\left[\frac{\mathrm{F}}{E^{*}}\right]^{\frac{1}{3}} \\
\frac{1}{E^{*}}=\frac{1-v_{p}^{2}}{E_{p}}+\frac{1-v_{g}^{2}}{E_{g}}
\end{gathered}
$$

where $F$ is the diametral force on the particle, $v_{g}, E_{g}, v_{p}, E_{p}$ are the Poisson's ratio and elastic modulus of the glass particle and the plate respectively. $a$ is the radius of the contact area between the particle and the flat plate and $R$ is the radius of the particle. 
The maximum tensile stress inside the volume of the particle is given by [20]:

$$
\sigma_{v}=\frac{2.8 F}{\pi d_{f}^{2}}
$$

$d_{f}$ is the distance between the loading points at the instance of failure. For particles with small deformation before failure, $d_{f}$ can be assumed to be equal to the diameter of the particle.

The surface crack initiating near the contact area is assumed to be an edge crack in a semiinfinite medium. The stress intensity factor can be written as:

$$
K_{I}=1.12 \sigma_{c} \sqrt{\pi c}
$$

where $c$ is the size of the critical flaw. Substituting the expression for the stress at the contact yields:

$$
F_{c}=\frac{9 \pi \sqrt{\pi} K_{I}^{3} R^{2}}{2(1.12)^{3}(1-2 v)^{3}\left(E^{*}\right)^{2} c^{\frac{3}{2}}}
$$

The volume crack initiating inside the particle is assumed to be a crack in the center of an infinite plate. The stress intensity factor is given by:

$$
K_{I}=\sigma_{v} \sqrt{\pi c}
$$

Substituting the expression for the stress at the contact gives:

$$
F_{v}=\frac{4 \sqrt{\pi} K_{I} R^{2}}{2.8 \sqrt{c}}
$$


Assuming the size of flaws is uniform through the volume and on the surface, the ratio of critical forces for initiation of fracture at contact and inside the volume is given by:

$$
\frac{F_{c}}{F_{v}}=\frac{\left(7.04 K_{I}^{2}\right)}{(1-2 v)^{3}\left(E^{*}\right)^{2} c}
$$

This formula is used to calculate the flaw sizes present in the glass particle based on the previously reported material properties and the experimentally observed forces required to initiate fracture at the contact and inside the volume respectively.

The material properties for glass particles are given by [35]

$$
\begin{gathered}
E_{g}=83 \mathrm{GPa} \\
v_{g}=0.2 \\
K_{I}=1.28 \mathrm{MPa} \mathrm{m}^{\frac{1}{2}}
\end{gathered}
$$

From the experimentally recorded ratios of forces for fracture at contact and inside the volume, the average flaw sizes in the particles are between $25.8 \mathrm{~nm}$ and $69.3 \mathrm{~nm}$. 


\section{Tables:}

Table 1: Material properties used for the Hertzian relations:

\begin{tabular}{|l|l|l|}
\hline Material & Young's modulus (GPa) & Poisson's ratio $(v)$ \\
\hline Steel & 210 & 0.3 \\
\hline Glass [35] & 83 & 0.2 \\
\hline Aluminum & 70 & 0.3 \\
\hline
\end{tabular}




\section{Figure Captions:}

Figure 1: Schematic of the experimental setup

Figure 2: Schematic of particle arrangement for (a) single particle experiments, (b) two particle experiments, (c) three particle experiments, and (d) five particle experiments. Note that the maximum number of particle-particle contacts increase from zero to one to two to four as the number of particles are increased from one to five respectively.

Figure 3: High speed X-ray images and corresponding force-displacement data for a representative single untreated glass particle compression experiment. The scale bar in the top right corner represents $500 \mu \mathrm{m}$. Recording speed: 2 million fps, exposure time: $200 \mathrm{~ns}$. The multimedia video provides images for the complete compression sequence.

Figure 4: High speed X-ray images and corresponding force-displacement data for a representative single annealed glass particle compression experiment. The scale bar in the top right corner represents $500 \mu \mathrm{m}$. Recording speed: 2 million fps, exposure time: $200 \mathrm{~ns}$. The multimedia video provides images for the complete compression sequence.

Figure 5: High speed X-ray images and corresponding force-displacement data for a representative two untreated glass particle compression experiment. The scale bar in the top right corner represents $500 \mu \mathrm{m}$. Recording speed: 5 million fps, exposure time: $110 \mathrm{~ns}$. The multimedia video provides images for the complete compression sequence.

Figure 6: High speed X-ray images and corresponding force-displacement data for a representative two annealed glass particle compression experiment. The scale bar in the top right corner represents $500 \mu \mathrm{m}$. Recording speed: 2 million fps, exposure time: $200 \mathrm{~ns}$. The multimedia video provides images for the complete compression sequence.

Figure 7: High speed X-ray images for a representative three glass particle compression experiment. The scale bar in the top right corner represents $500 \mu \mathrm{m}$. Recording speed: 2 million fps, exposure time: 200 ns. The multimedia video provides images for the complete compression sequence.

Figure 8: High speed X-ray images for a representative five glass particle compression experiment. The scale bar in the top right corner represents $500 \mu \mathrm{m}$. Recording speed: 2 million fps, exposure time: 200 ns. The multimedia video provides images for the complete compression sequence. 


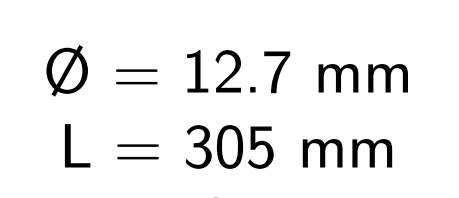

$\varnothing=12.7 \mathrm{~mm}$

$\mathrm{L}=1372 \mathrm{~mm}$

Bar Striker
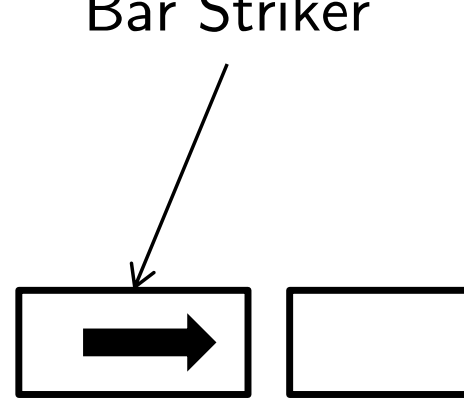

Objective Lens

$5 X$ Incident Bar

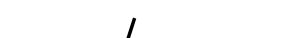

Shimadzu HPV-X

High speed

camera

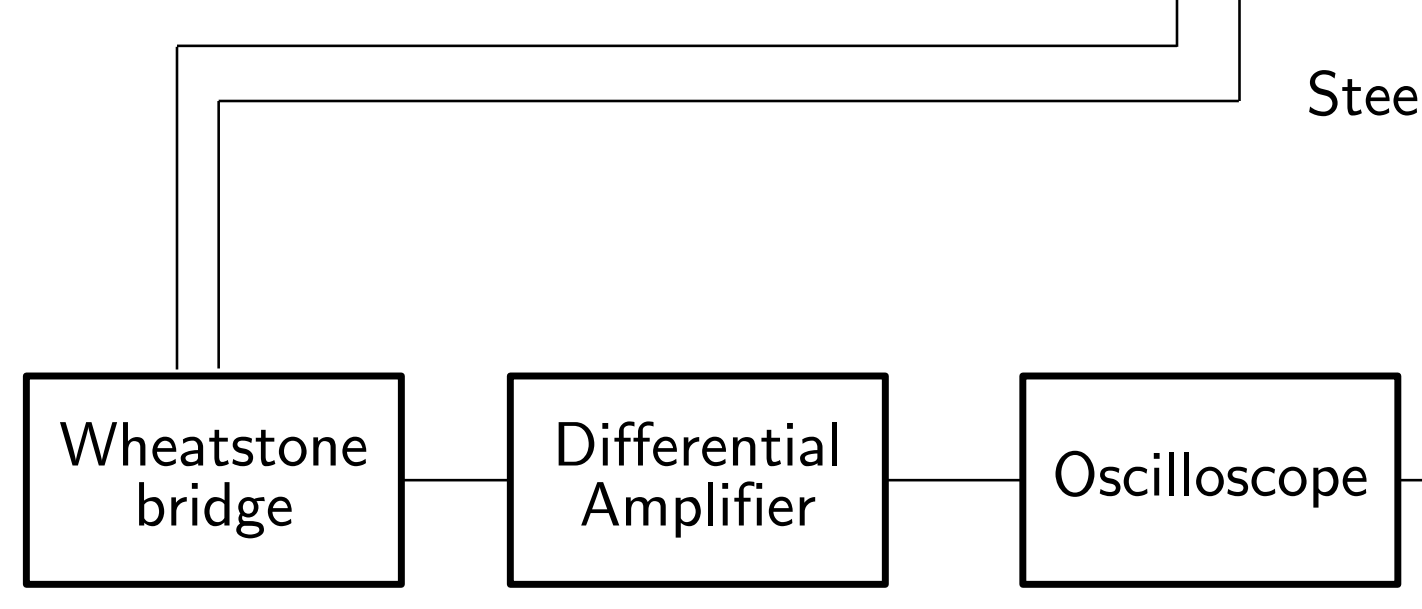

Strain gauges

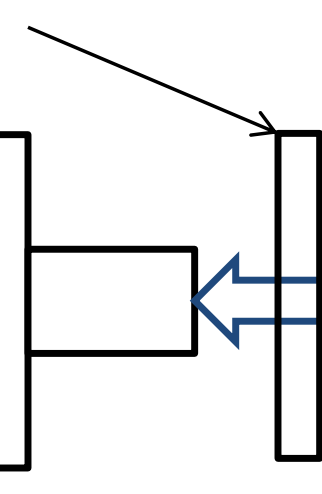

Scintillator

Mirror
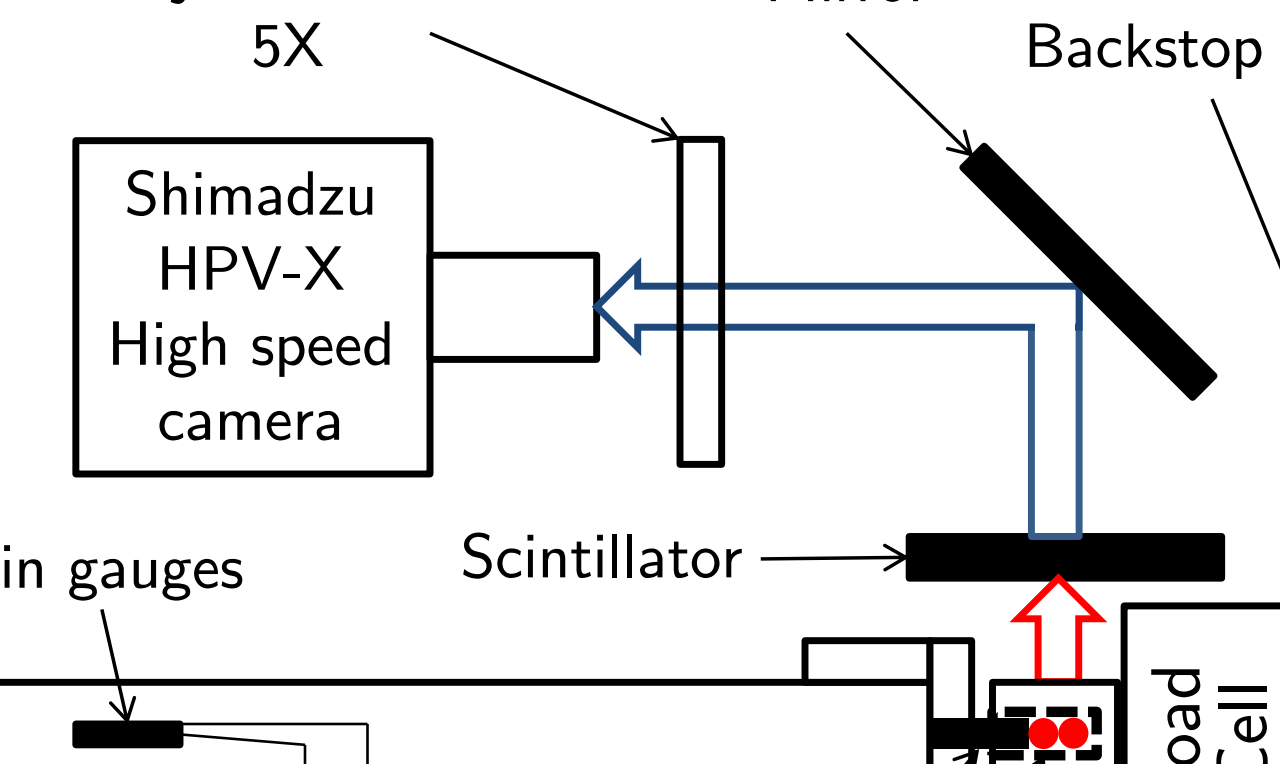


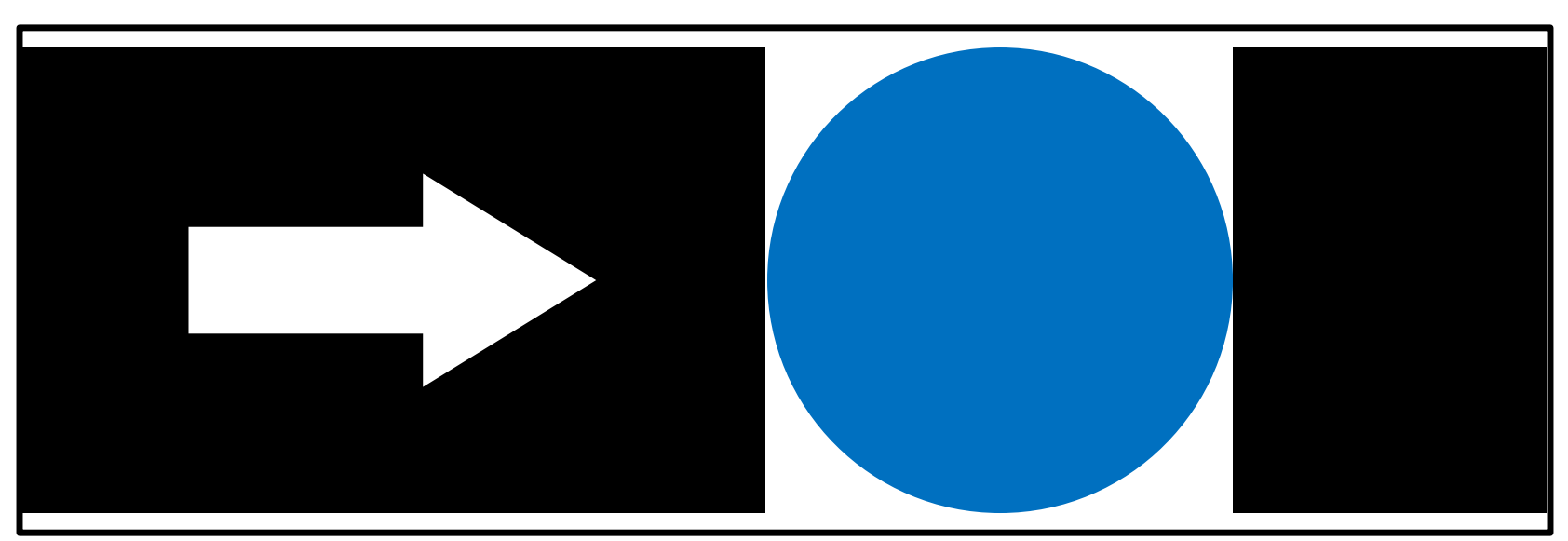

(a)

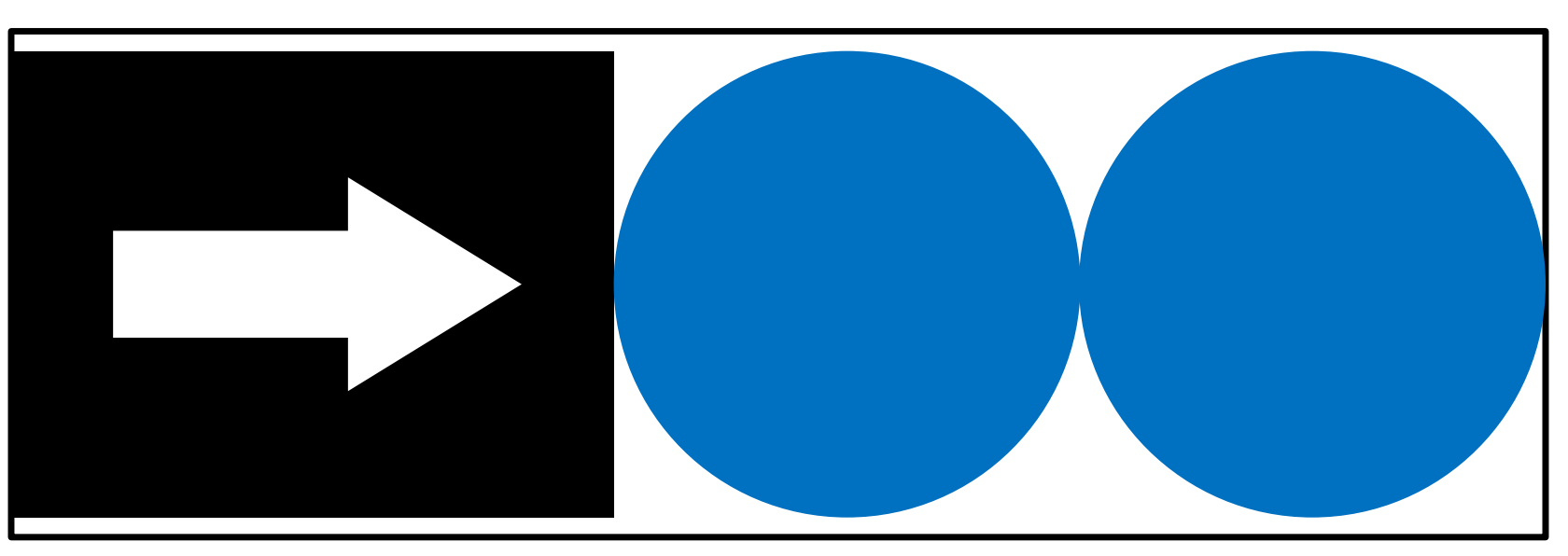

(b)

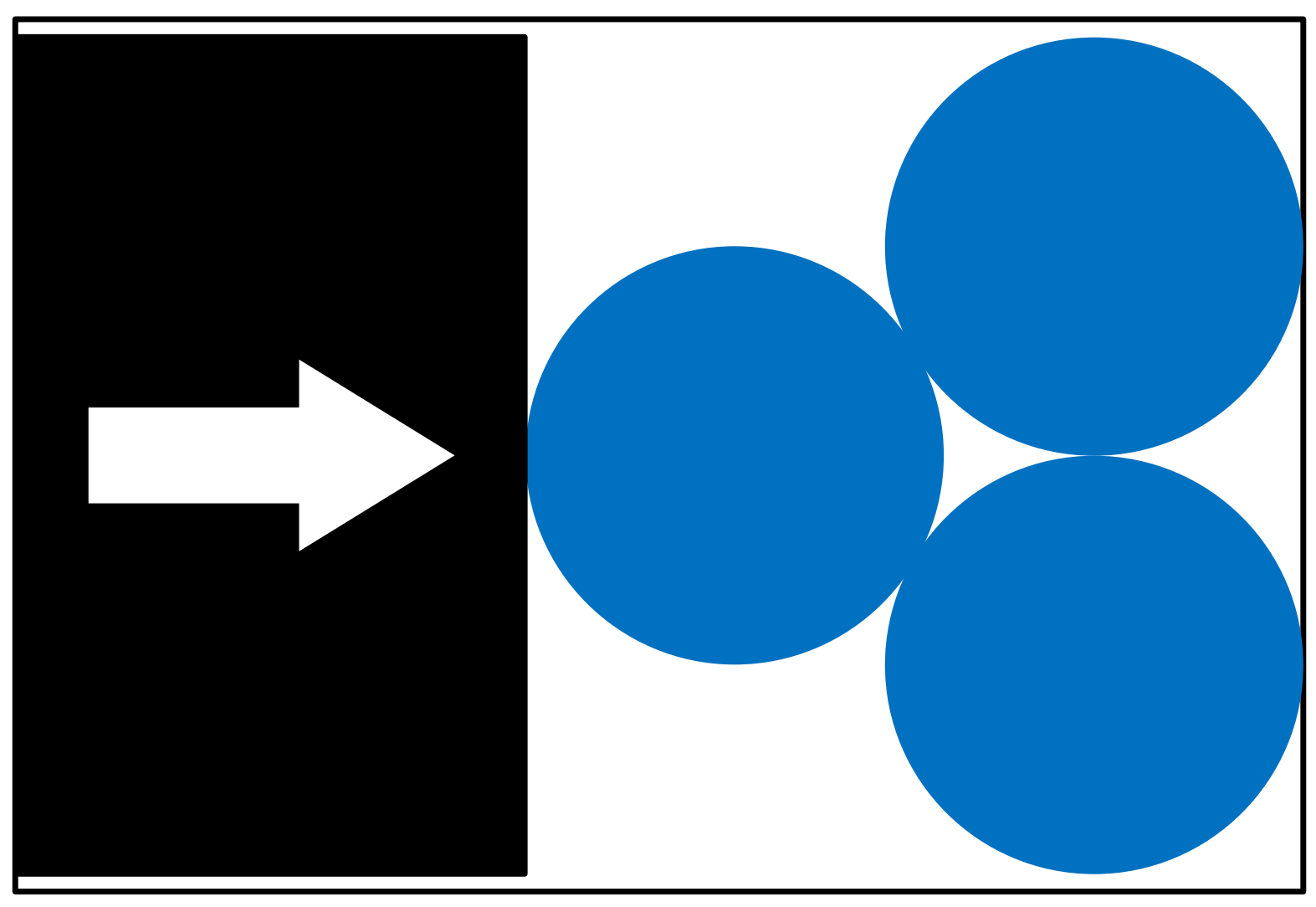

(c)

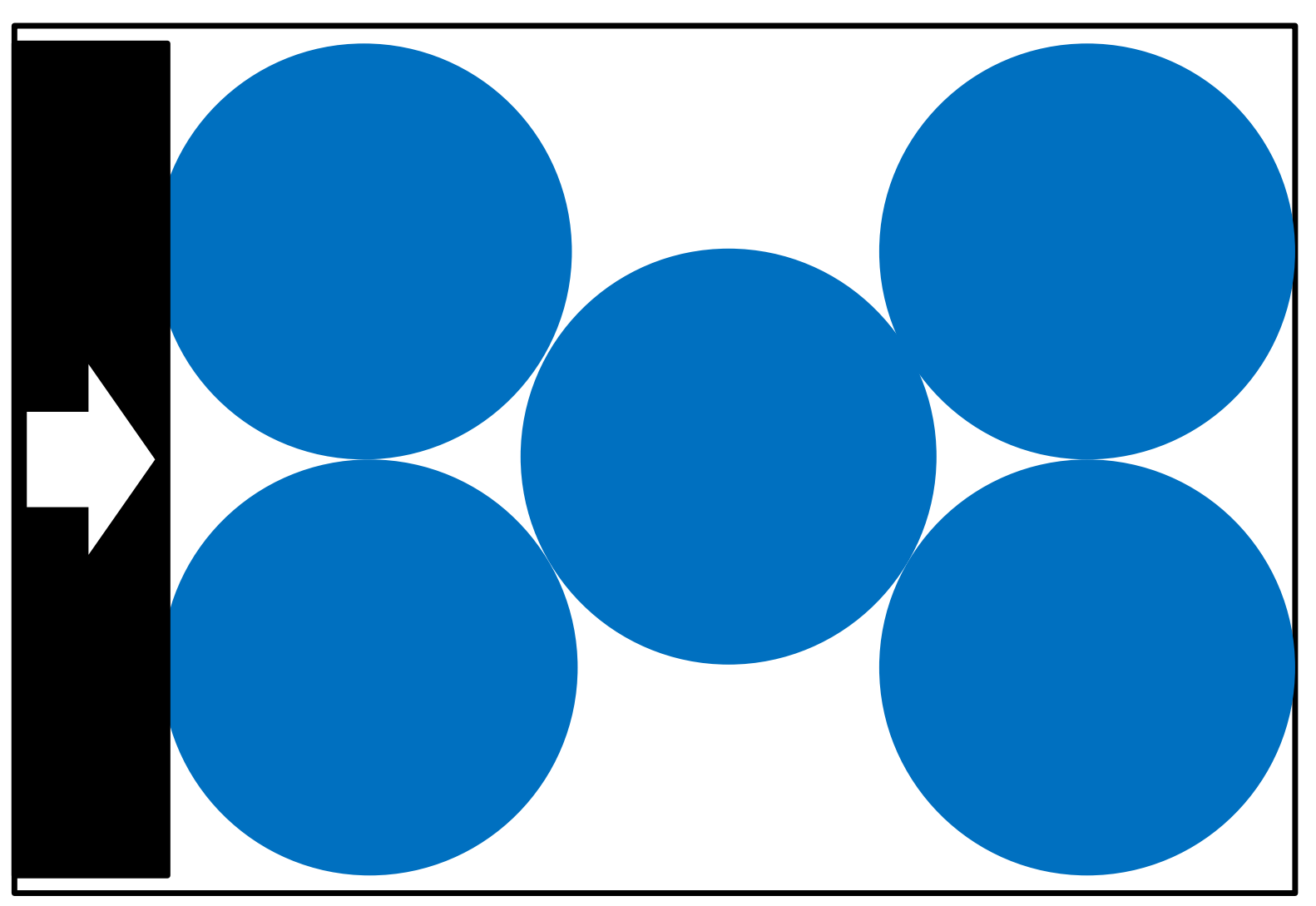

(d) 


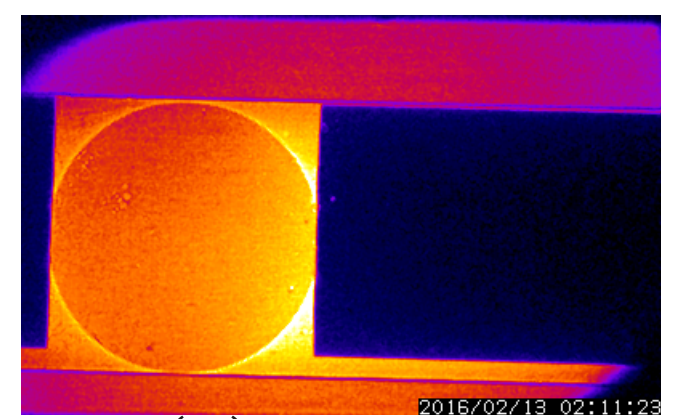
(1) $\mathrm{t}=0 \mu \mathrm{s}$

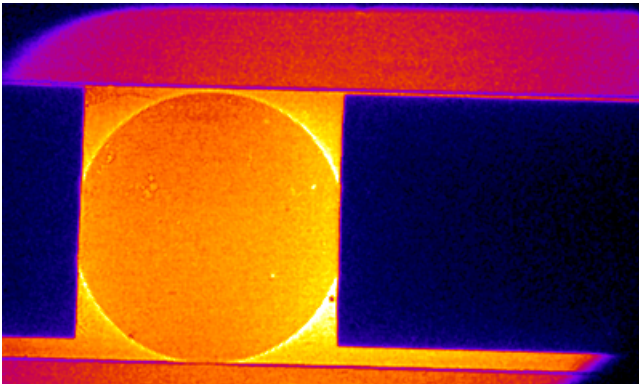

(2) $t=30 \mu \mathrm{s}$

(3) $t=60 \mu \mathrm{s}$
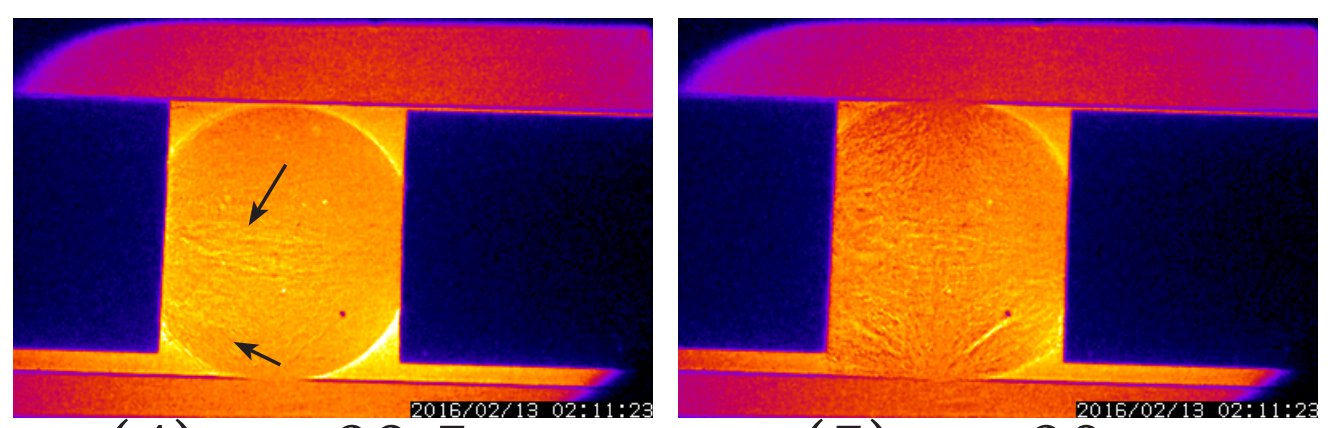

(4) $\mathrm{t}=89.5 \mu \mathrm{s}$

(5) $t=90 \mu \mathrm{s}$
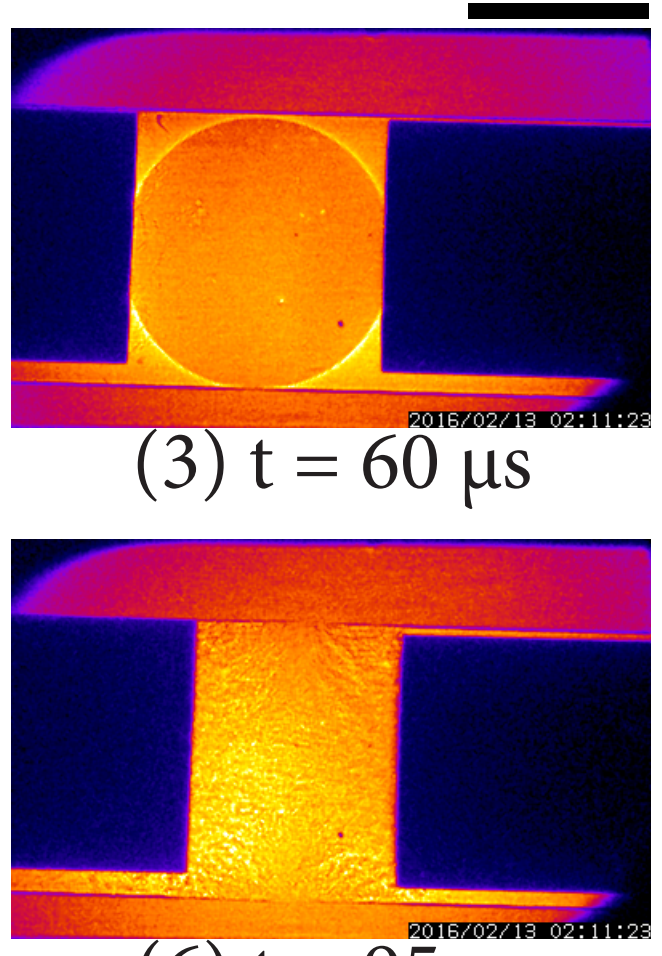

(6) $t=95 \mu \mathrm{s}$

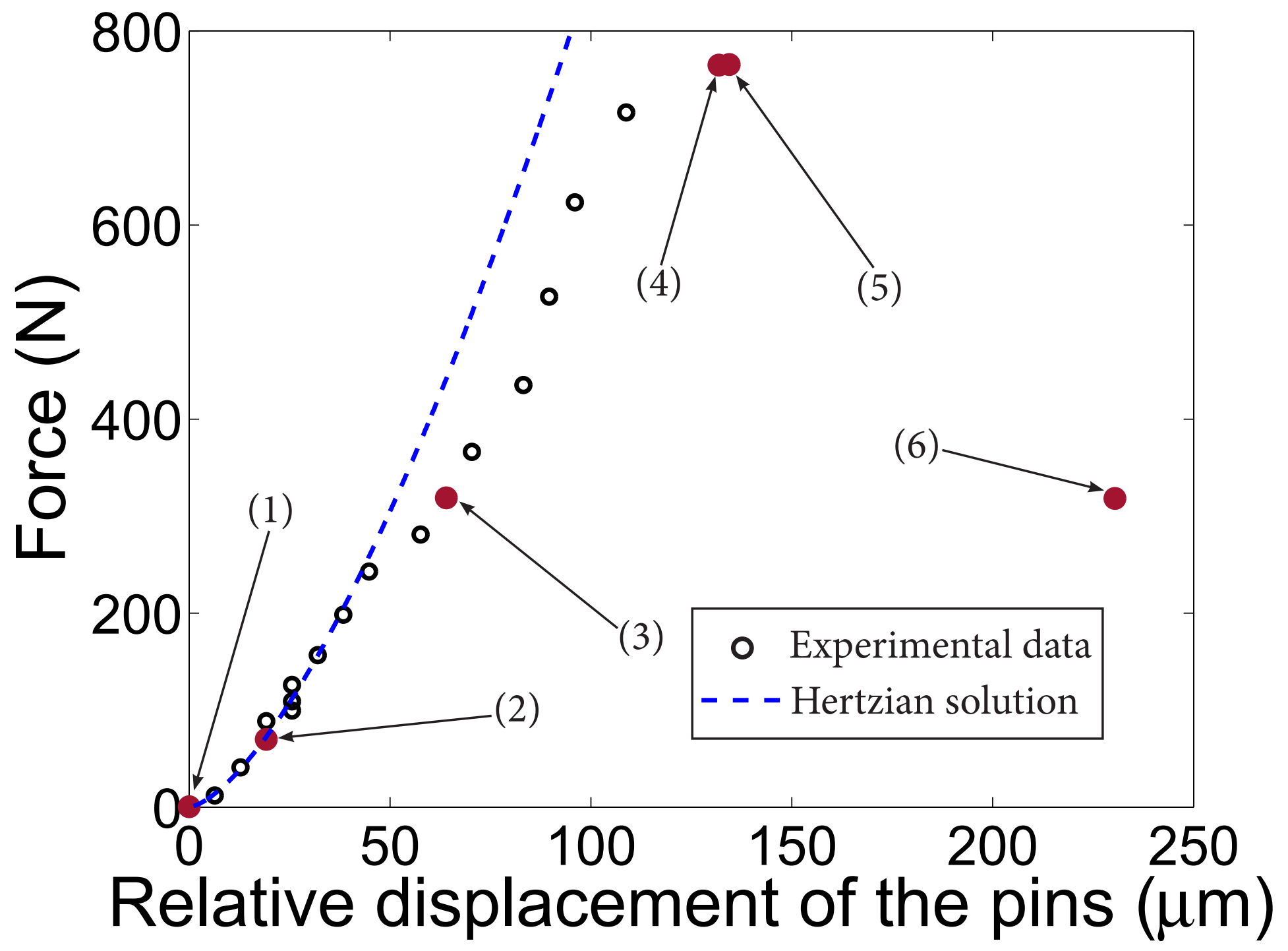




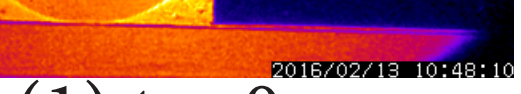

(1) $t=0 \mu \mathrm{s}$

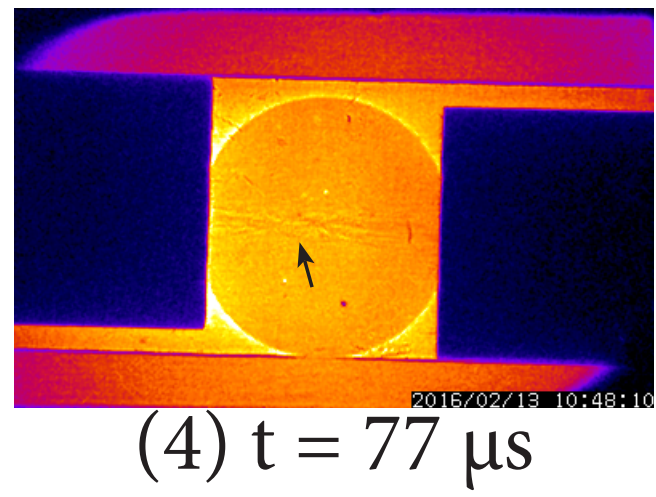

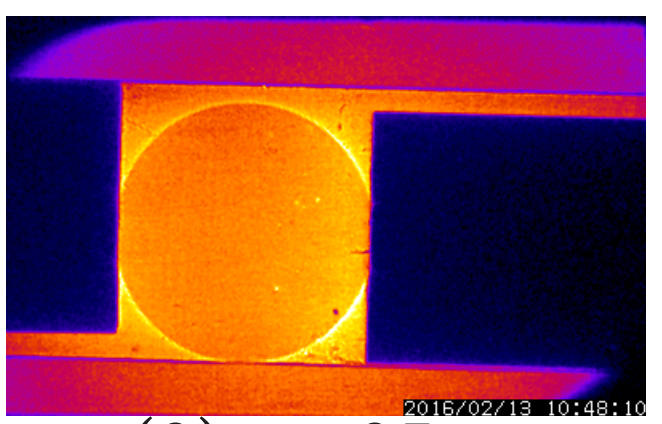

(2) $t=25 \mu \mathrm{s}$

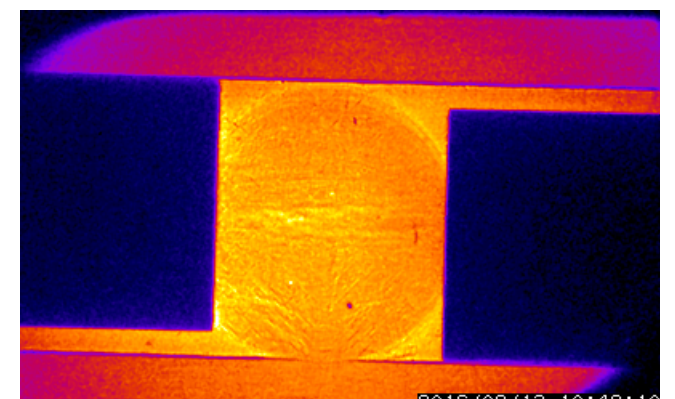

(5) $\mathrm{t}=77.5 \mu \mathrm{S}$

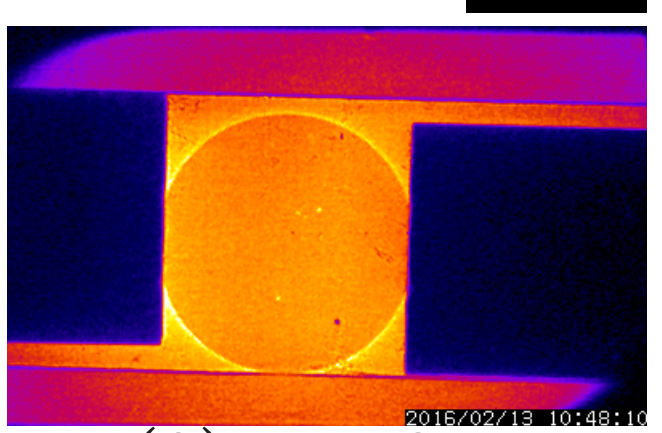

(3) $t=50 \mu \mathrm{s}$

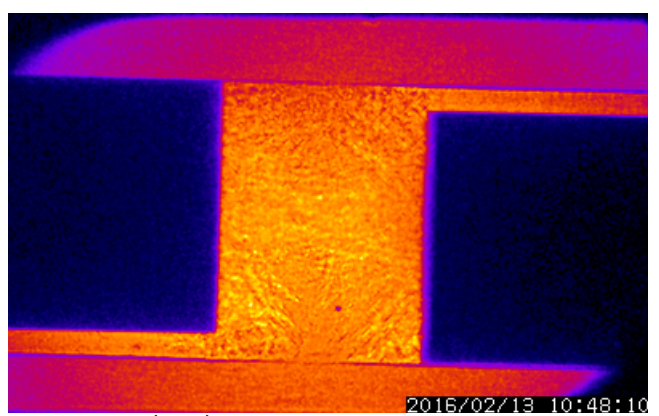

(6) $t=80 \mu \mathrm{s}$

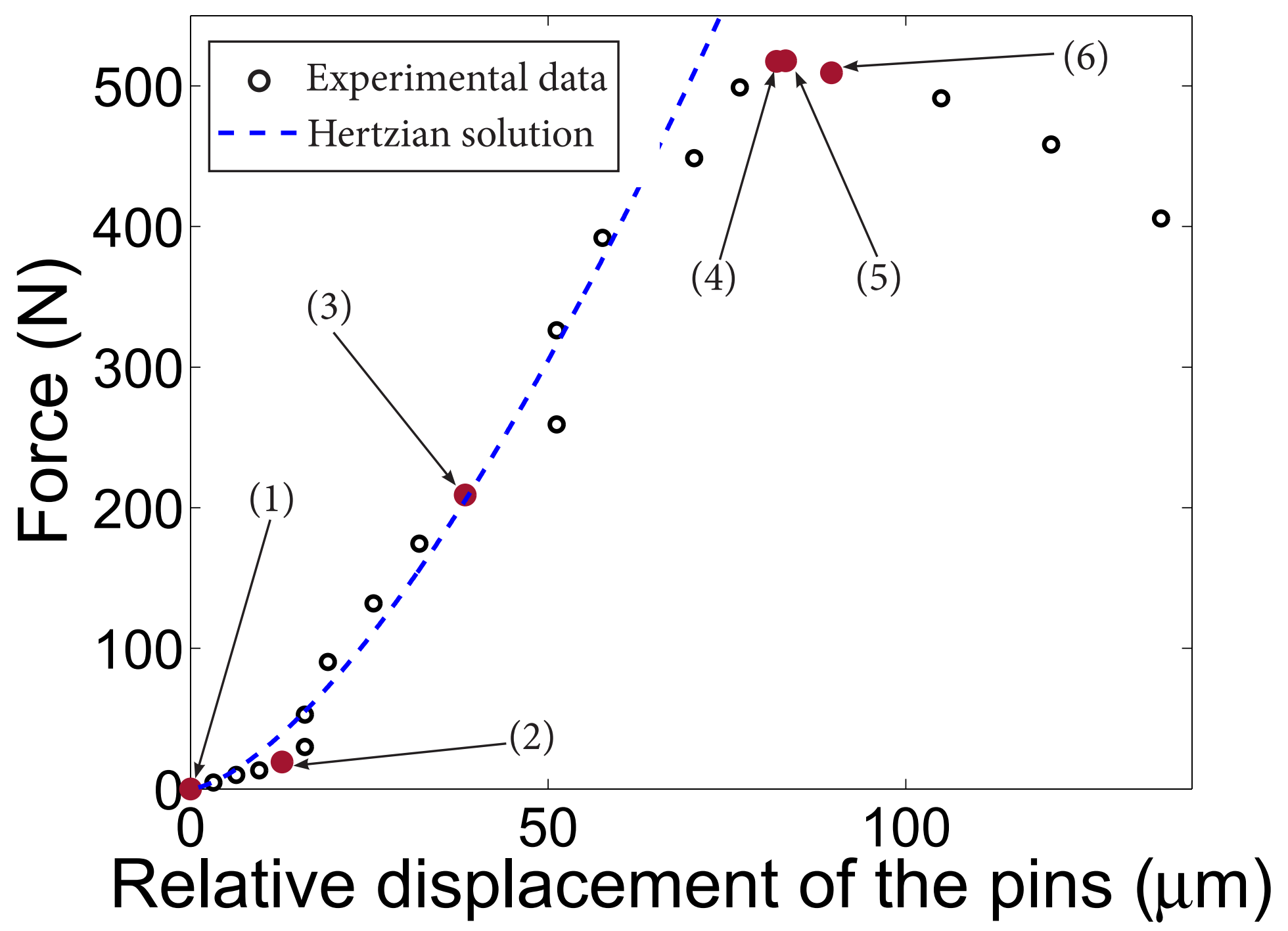




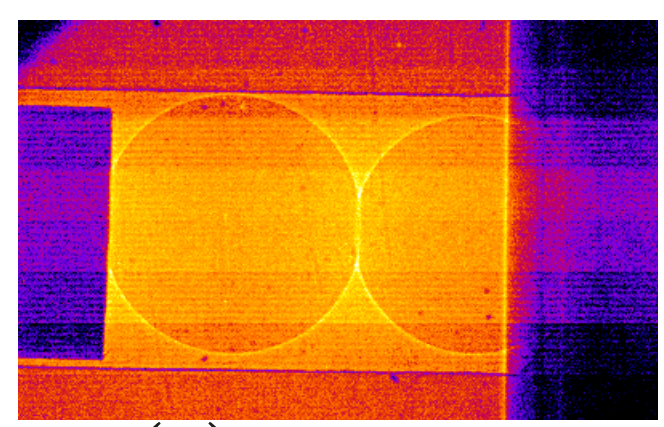

(1) $t=12 \mu \mathrm{s}$

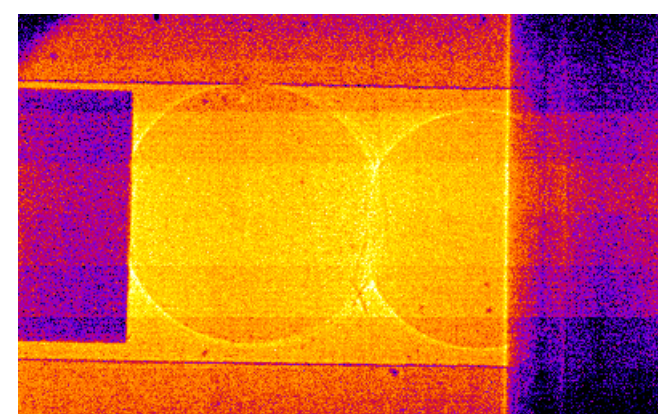

(4) $\mathrm{t}=33.8 \mu \mathrm{s}$ 400

- Experimental data

- - - Hertzian solution

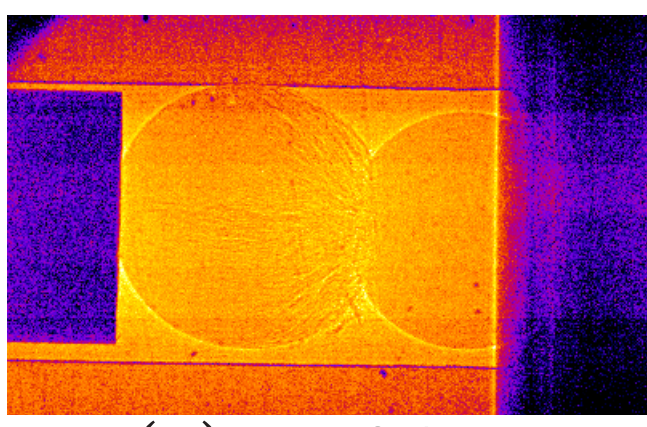

(5) $t=34 \mu s$

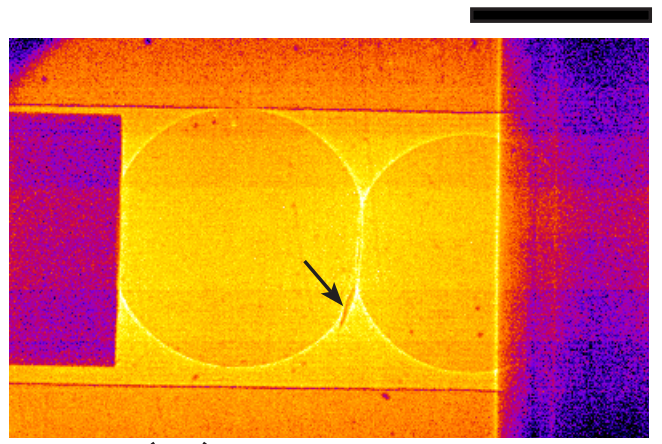

(3) $t=32 \mu \mathrm{s}$

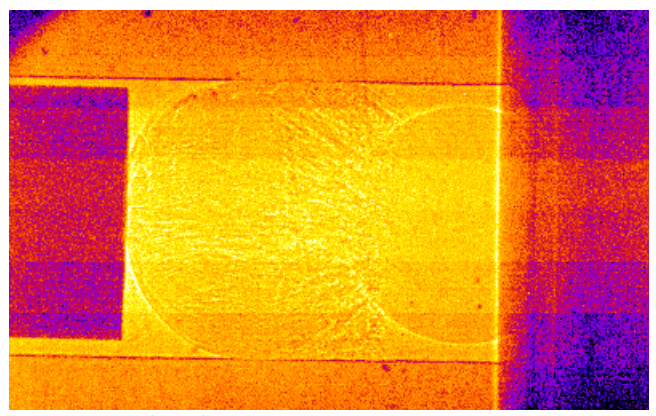

(6) $t=35 \mu s$

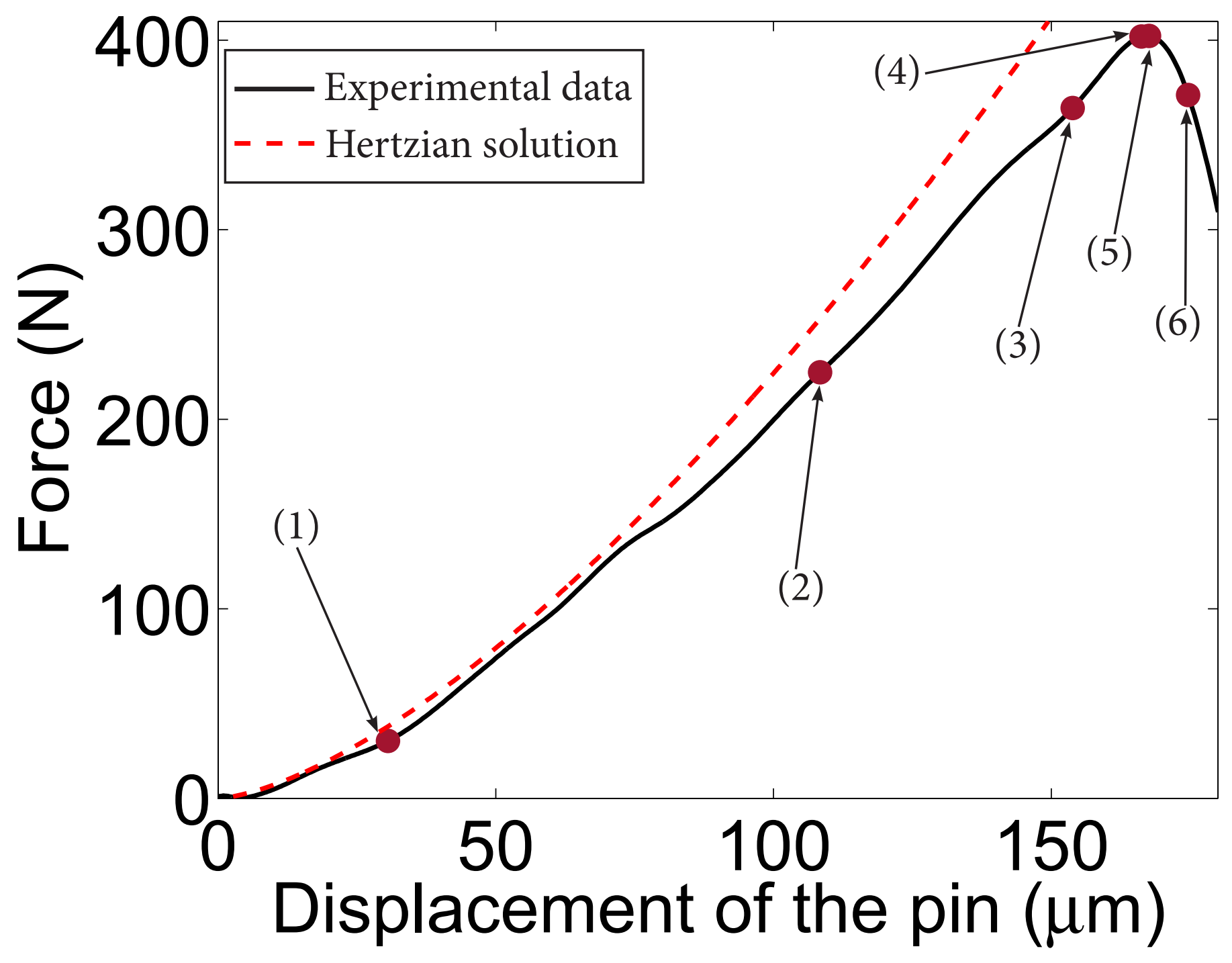




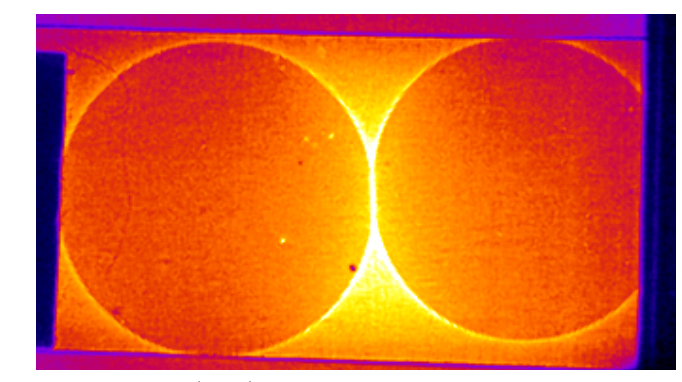

(1) $\mathrm{t}=0 \mu \mathrm{s}$

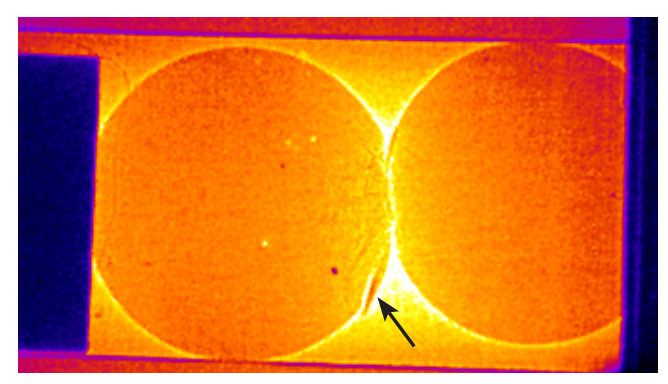

(4) $t=28 \mu \mathrm{s}$

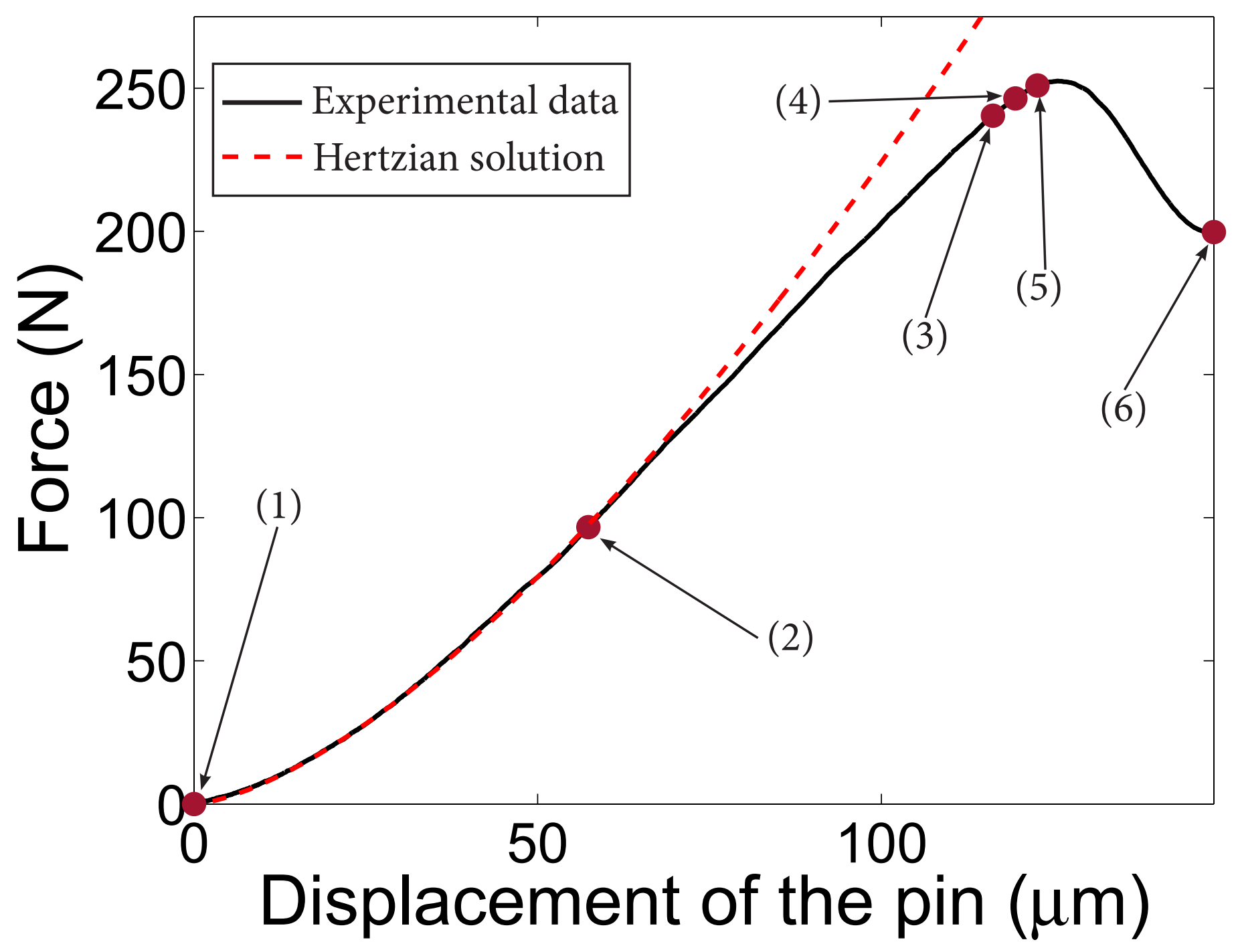

(2) $t=18 \mu \mathrm{s}$

(3) $t=27.5 \mu \mathrm{s}$

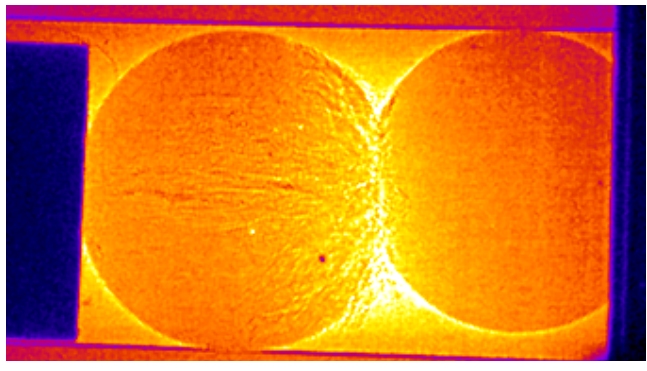

(5) $t=28.5 \mu \mathrm{s}$

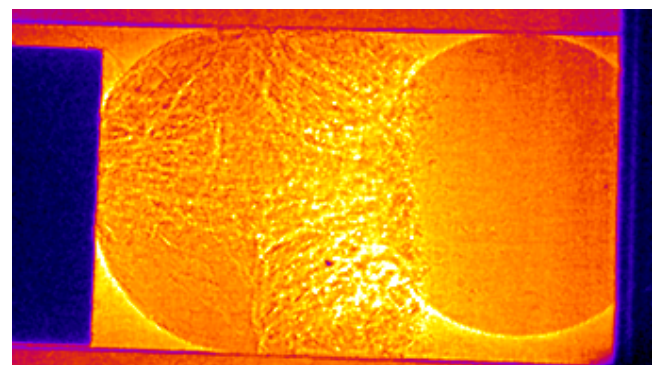

(6) $t=30 \mu s$ 


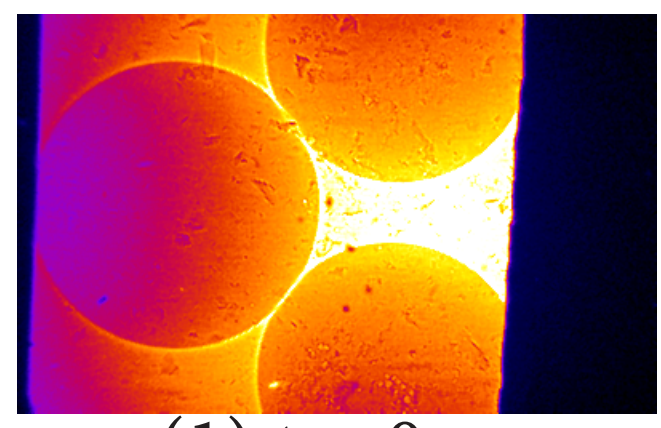

(1) $t=0 \mu s$

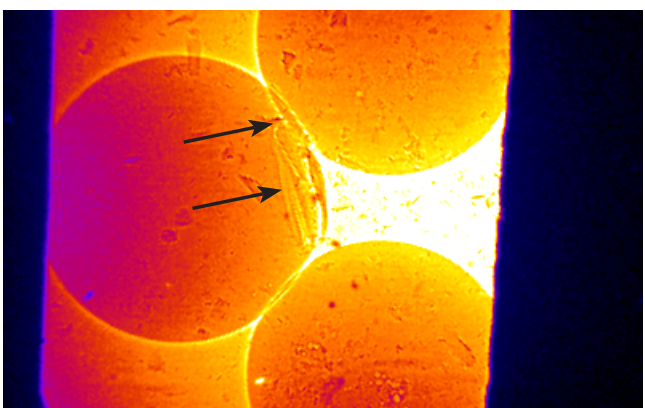

(2) $t=22.5 \mu s$
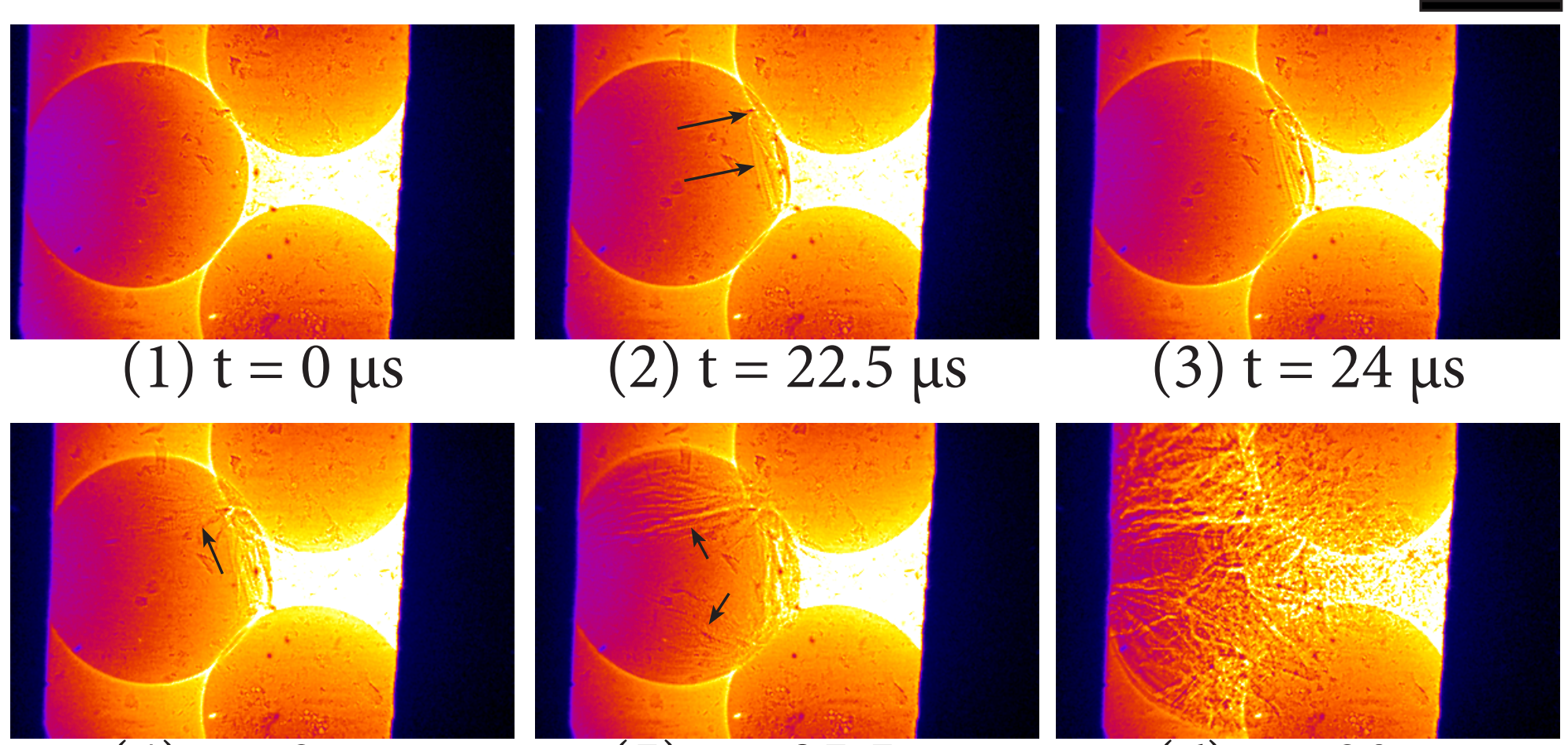

(4) $t=25 \mu \mathrm{s}$

(5) $t=25.5 \mu s$

(3) $t=24 \mu s$

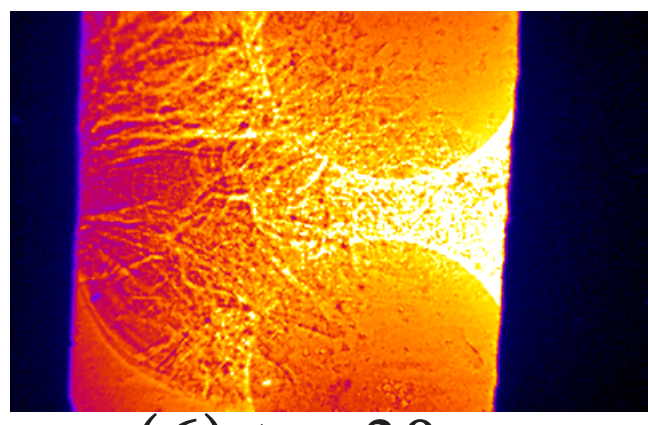

(6) $t=30 \mu s$ 


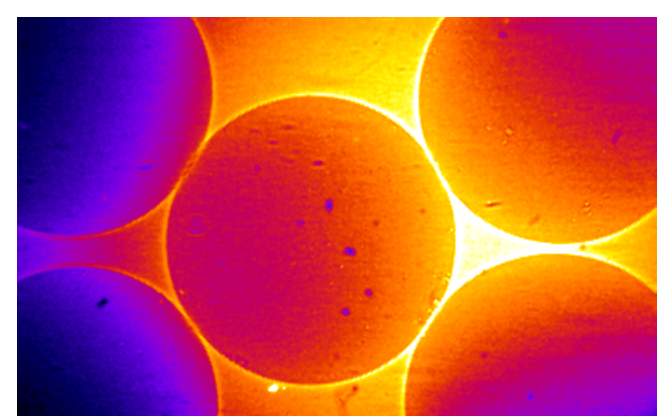

(1) $\mathrm{t}=0 \mu \mathrm{s}$

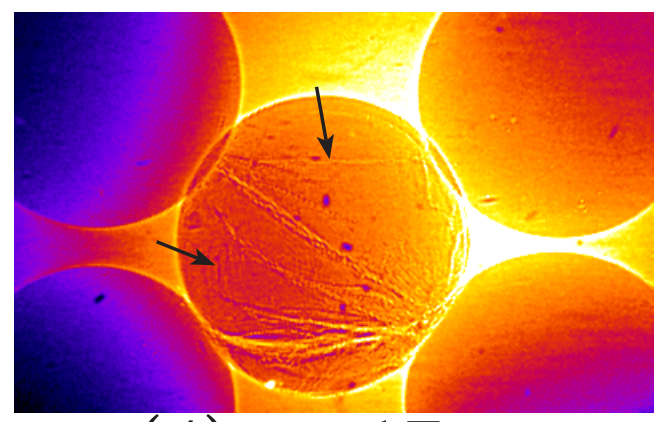

(4) $\mathrm{t}=17 \mu \mathrm{s}$

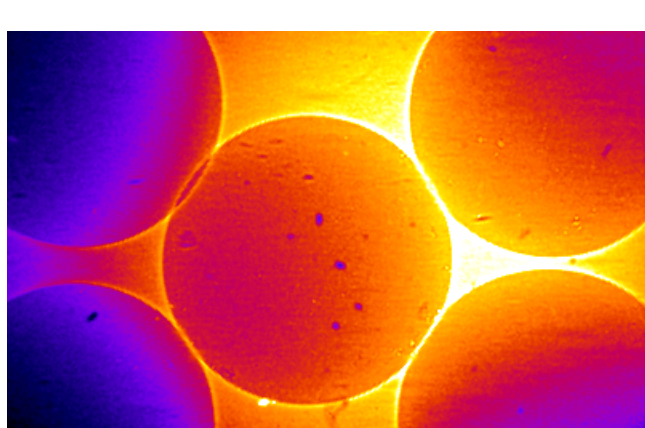

(2) $\mathrm{t}=10 \mu \mathrm{s}$

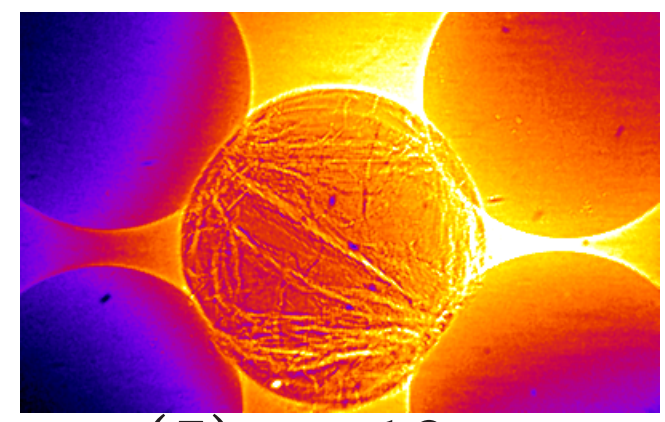

(5) $\mathrm{t}=18 \mu \mathrm{s}$

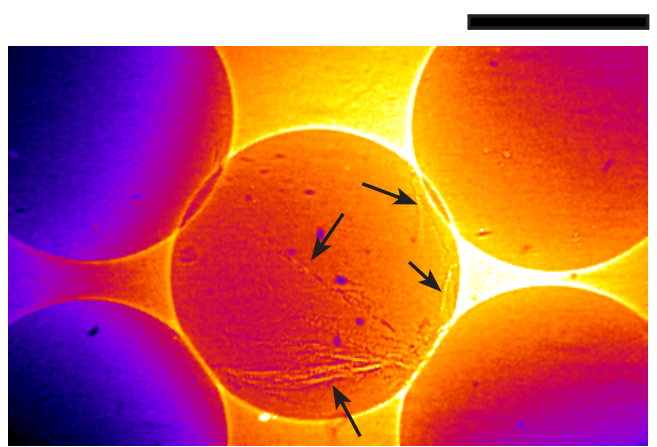

(3) $\mathrm{t}=16.5 \mu \mathrm{s}$

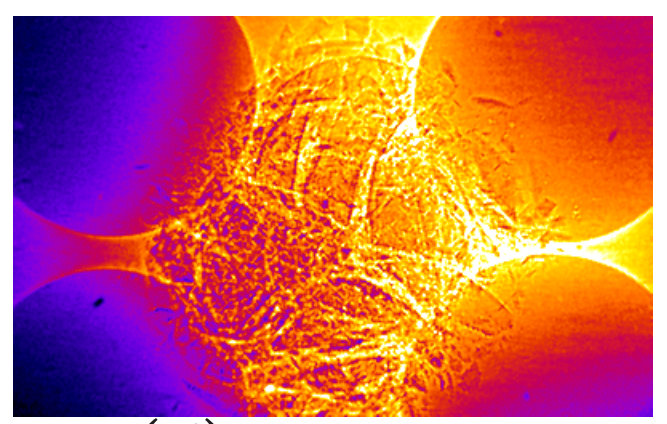

(6) $t=23 \mu \mathrm{s}$ 

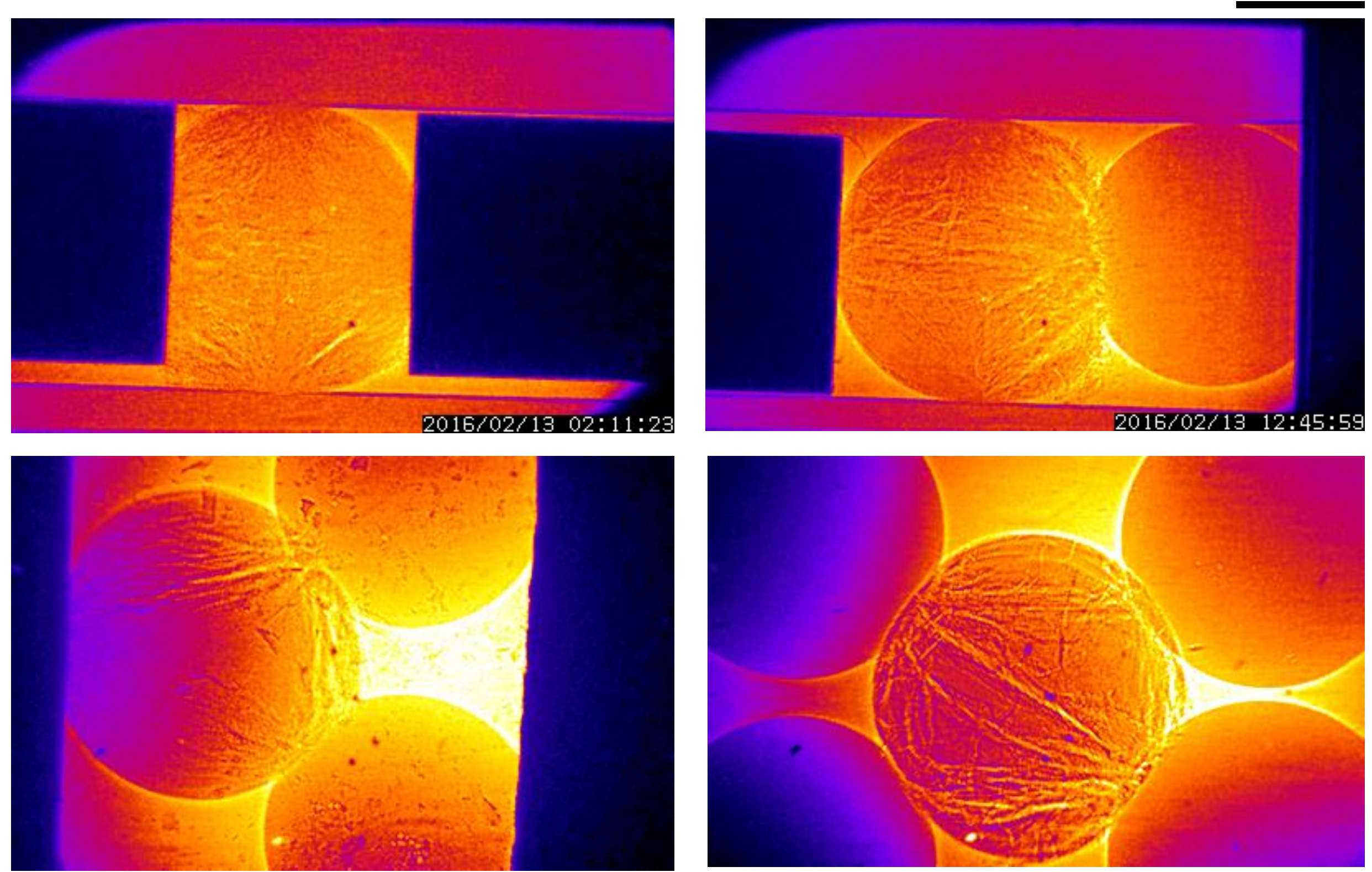

The fracture initiation mode of glass particles under dynamic compression was observed to depend strongly on the contact conditions. However, the final mechanism was observed to be explosive fragmentation for all experiments. (Scale bar in top right corner $=500 \mu \mathrm{m}$ ) 